\title{
Les Komis de l'Ižma et les Pomors
}

Deux modèles de transformations culturelles

The Izhma Komi and the Pomors: two models of cultural change

КОМИ-ИЖЕМЦЫ И ПОМОРЫ : ДВЕ МОДЕЛИ КУЛЬТУРНЫХ ТРАНСФОРМАЦИЙ

Jurij P. Šabaev

Traducteur : Eva Toulouze

\section{OpenEdition}

\section{Journals}

Édition électronique

URL : https://journals.openedition.org/efo/165

DOI : $10.4000 /$ efo. 165

ISSN : 2275-1947

Éditeur

INALCO

Édition imprimée

Date de publication : 1 janvier 2011

ISBN : 978-2-296-96641-3

ISSN : 0071-2051

Référence électronique

Jurij P. Šabaev, «Les Komis de l'Ižma et les Pomors », Études finno-ougriennes [En ligne], 43 | 2011, mis en ligne le 20 mars 2014, consulté le 08 juillet 2021. URL : http://journals.openedition.org/efo/165 ; DOI : https://doi.org/10.4000/efo.165

Ce document a été généré automatiquement le 8 juillet 2021

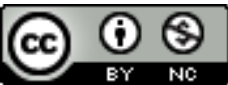

Études finno-ougriennes est mis à disposition selon les termes de la Licence Creative Commons Attribution - Pas d'Utilisation Commerciale 4.0 International. 


\title{
Les Komis de l'Ižma et les Pomors
}

\author{
Deux modèles de transformations culturelles \\ The Izhma Komi and the Pomors: two models of cultural change \\ КОМИ-ИЖЕМЦЫ И ПОМОРЫ : ДВЕ МОДЕЛИ КУЛЬТУРНЫХ ТРАНСФОРМАЦИЙ
}

\author{
Jurij P. Šabaev
}

Traduction : Eva Toulouze

\section{Introduction}

1 Les Komis de l'Ižma et les Pomors présentent un intérêt évident pour la recherche en anthropologie. Cet intérêt ne tient d'ailleurs aucunement à la dimension archaïque de leurs traditions culturelles, qui s'est en grande partie perdue, mais à la mémoire historique du groupe et à son identité ethnique (ou locale), qui forment le fondement de leur positionnement culturel. Dans les deux cas, nous pouvons parler de réidentification: certes, l'ethnonyme des Komis de l'Ižma (Iz'vatas) avait été pris en compte par les statistiques officielles en 1926 et puis, après un long intervalle, en 1989, mais seulement en tant que variante de l'ethnonyme Komis (renvoyant aux KomisZyriènes) ; les Pomors, pour leur part, figurent dans les documents du recensement de 1989, mais également en qualité de variante de l'ethnonyme primaire russe (Sokolovskij, 2004, pp. 225-226).

2 Le recensement de 2002 a permis un large débat dans le corps social sur la restitution aux groupes locaux de leurs dénominations antérieures et sur la manière dont elles permettent de déterminer des groupes réellement existants. Il se distinguait également des précédents par le fait que dans sa préparation, les ethnologues de Russie ont pu obtenir l'inclusion dans les documents officiels de l'éventail le plus large possible de «catégories de prise en compte de la composition ethnique de la population ». Cela a permis de relever l'existence de 6500 Pomors et de 16500 Komis de l'Ižma : cela veut dire que des groupes considérables ont préféré s'identifier par des indicateurs ethniques non point généraux, mais locaux. Cependant, le seul acte formel de la part d'un groupe de citoyens de s'identifier à l'aide de tel ou tel marqueur ethnique ne 
permet pas à lui seul d'attester l'évidence de l'existence d'un ensemble. Qui plus est, dans la plupart des cas, la prise en compte des ethnonymes locaux n'amène pas les personnes les choisissant pour s'identifier à être considérées comme ressortissant de ces communautés particulières: elles sont incluses dans les effectifs d'un groupe ethnique plus large (les Pomors parmi les Russes, les Iz'vatas parmi les Komis, etc.).

C'est pourquoi, pour prendre en compte ce que représente ce groupe de personnes se désignant par le même définisseur ethnique, il est important d'examiner un ensemble de phénomènes culturels : la solidarité interne, l'idéologie de groupe, le contenu de l'identité, la capacité collective à formuler et à exprimer des intérêts communs et d'autres facteurs qui caractérisent la situation culturelle au sein du groupe. La méthode la plus adéquate pour étudier cette dynamique, me semble-t-il, repose sur un schéma tripartite - processus, participants, circonstances - sur la base de l'analyse de discours proposée par Norman Fairclough (Fairclough, 2003), qui nous semble totalement pertinente dans ce cas précis. Plus généralement, nous nous rapprochons de la vision constructiviste de la nature de l'ethnicité (Theories, 1996), sans pour autant nous enfermer dans cette approche méthodologique.

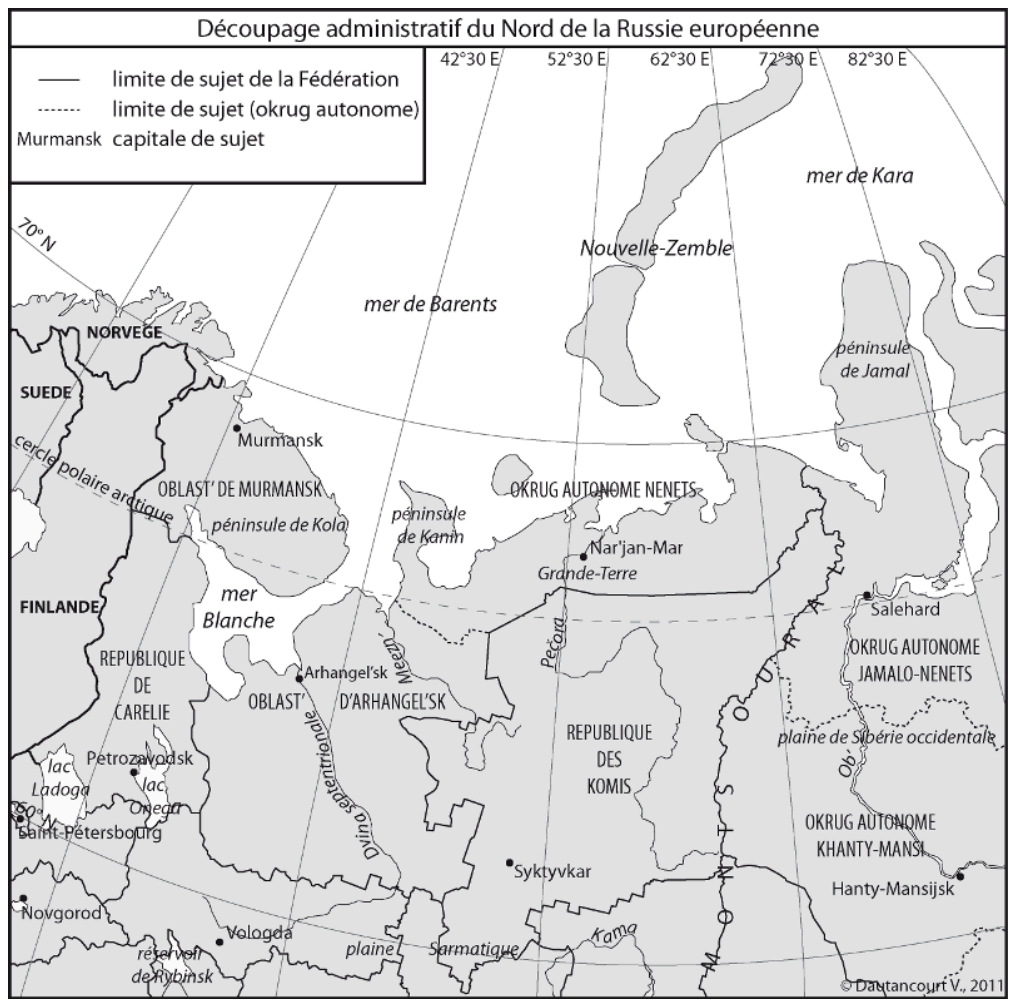



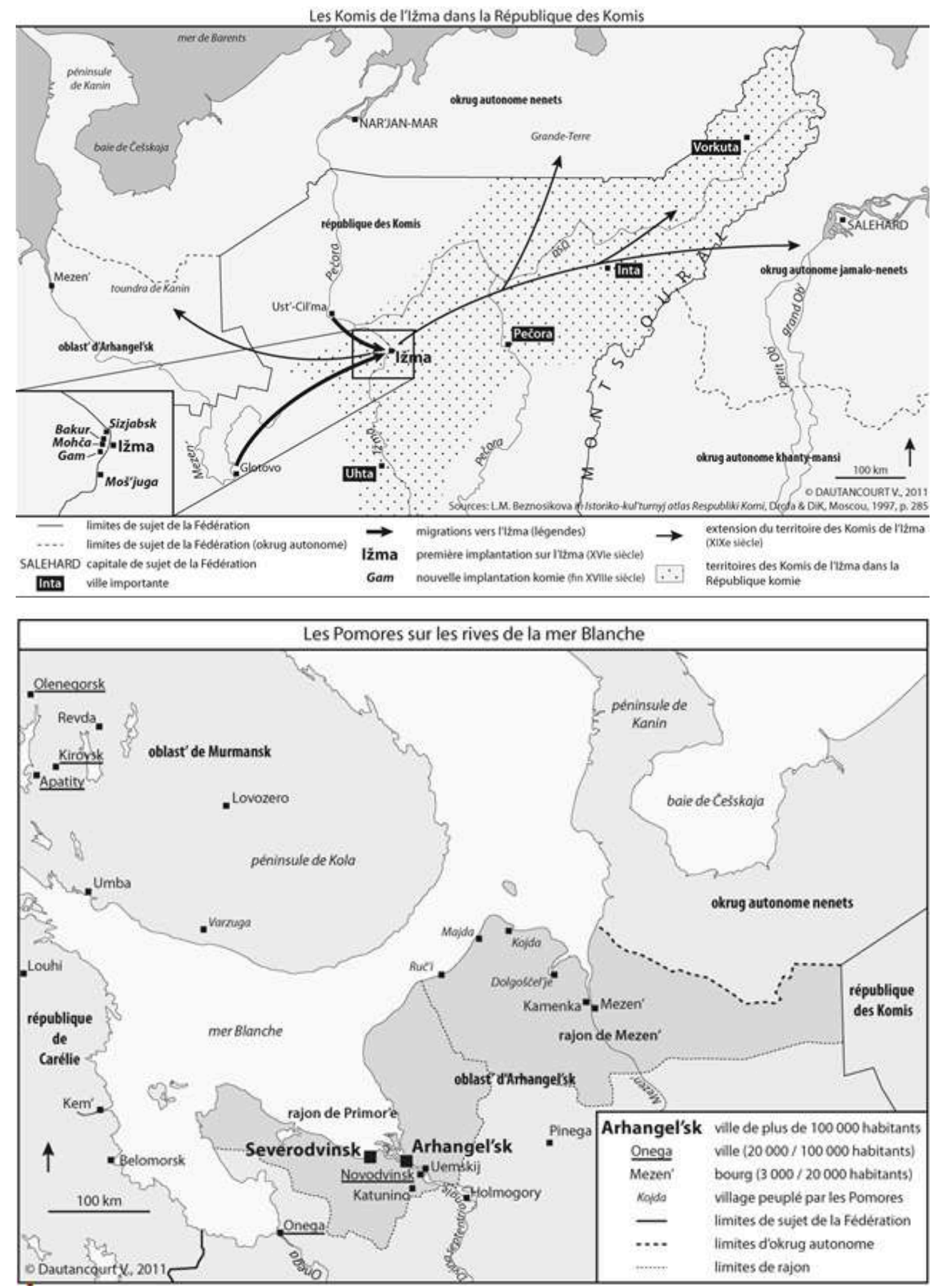

\section{Histoire de la formation des groupes ethniques}

Parmi les groupes ethnographiques au sein du peuple komi, ce sont les Komis de l'Ižma (Iz'vatas) qui ont la culture la plus originale. La formation de ce groupe remonte à la fin du XVI ${ }^{\mathrm{e}}$ siècle. C'est entre 1568 et 1575 qu'a été constituée sur l'Ižma, un affluent de la Pečora, la sloboda du même nom. D'après les légendes, ses fondateurs étaient des migrants en provenance de la sloboda de Glotovo sur le haut Mezen' et de la sloboda d'Ust'-Cil'ma, fondée un quart de siècle plus tôt par Ivaška Latska, de Novgorod. Longtemps, la sloboda d'Ižma a été l'unique habitat komi dans le bas cours de la Pečora ; 
ce n'est qu'à la fin du XviII siècle qu'autour d'elle ont commencé à surgir de nouveaux points habités : Mohča, Sizjabsk, Gam, Bakur, Mošjuga.

5 C'est aux $\mathrm{XVII}^{\mathrm{e}}$-XVIII ${ }^{\mathrm{e}}$ siècles que le processus de formation du groupe le plus septentrional des Komis arriva pratiquement à son terme. Compte tenu d'un mélange interethnique prolongé et d'influences mutuelles, les Komis de l'Ižma développèrent des traits physiques particuliers, un dialecte spécifique sur la base du komi avec des emprunts lexicaux au russe et au nenets, et ils transformèrent leur système économique traditionnel, se différenciant ainsi fondamentalement des autres groupes ethnographiques komis. Au $\mathrm{XIX}^{\mathrm{e}}$ siècle, les Komis de l'Ižma agrandirent considérablement leur territoire. Ils fondèrent toute une série d'agglomérations dans le cours moyen de la Pečora, occupèrent le bassin de l'Usa, un autre affluent de la Pečora, prirent pied dans les toundras de Kanin et de la Grande Terre ${ }^{1}$, franchirent l'Oural et fondèrent des villages sur l'ob; de plus, un groupe non négligeable alla s'installer dans la péninsule de Kola (Lašuk, 1960 ; Žerebcov, 1982 ; Konakov, 1991).

6 Les Komis de l'Ǐma fondaient leur économie sur l'élevage du renne. Ils en avaient entièrement emprunté les principes aux Nenets dès le xvIII siècle. Ces emprunts ne concernaient pas uniquement le mode de gestion de l'économie, mais l'ensemble du système culturel, y compris l'habillement, l'habitat transportable, voire le folklore. En même temps, les Komis de l'Ižma introduisirent dans ce système des éléments nouveaux: la surveillance continue des troupeaux à l'aide des chiens, l'élevage commercial à grande échelle dans la toundra de la Grande Terre; ils utilisaient d'ailleurs largement la production de l'élevage du renne dans les activités commerciales : commerce des peaux, production de chamois.

7 Les Komis de l'Ižma avaient une économie complexe, dans laquelle les différentes branches étaient complémentaires, tout le système étant de manière générale plutôt flexible et adapté à son environnement. En plus de l'élevage du renne, ils pratiquaient la chasse et la pêche, l'élevage et un peu d'horticulture, commerçaient dans tout le nord de la Russie et de la Sibérie occidentale, livraient leurs produits jusqu'à Moscou et à Saint-Pétersbourg. Aptes à la gestion, ils s'avérèrent plus efficaces économiquement que les Nenets de la toundra de la Grande Terre, voire que les Sames de la péninsule de Kola ou les Khantys. De plus, ils jouaient sur le marché un rôle de banquiers et de fournisseurs pour la production des exploitants nenets (mais aussi khantys et sames). Ils devinrent ainsi pour les Nenets, et en partie aussi pour les autres groupes, des concurrents dangereux et ceux-là se plaignaient souvent aux représentants des autorités d'être opprimés par les Komis de l'Ižma.

8 Les éleveurs de rennes komis de l'Ǐžma multiplient le nombre de têtes de leurs troupeaux, et dès le milieu des années 1830 les Nenets avaient perdu leur prédominance dans la toundra, ce dont témoigne indirectement le «Statut sur la gestion des Samoyèdes de l'uezd du Mezen' (gouvernement d'Arkhangelsk) », adopté en 1835. Conformément à ses dispositions, tous les « allogènes » faisant paître leurs rennes sur les pâturages situés dans les "territoires samoyèdes » devaient payer des droits de pâturage par bête. Les chercheurs ont également noté que dans les années 1840, la taille des troupeaux respectivement des Nenets et des Komis allait de 1 à 4, proportion qui est restée identique dans les années suivantes (Zyrjanskij, 2004, p. 154).

9 À l'instar des Komis de l'Ǐžma, les Pomors se sont conquis un créneau spécifique dans le Nord et ont réussi à mettre en place une économie efficace. 
10 En général, la représentation scientifique est que le groupe des Pomors s'est formé il y a plusieurs siècles. La plupart des chercheurs soulignent que la dénomination du groupe (Pomors) est apparue dès le XII siècle (Gemp, 2005) et que c'est à cette époque-là que s'est activé le processus de sa formation. Mais V. Anufriev fait remarquer à juste titre que la première mention de la région « Pomor'e » (en russe : « maritime») remonte à 1459, et que l'ethnonyme pomor n'apparaît que dans la deuxième moitié du xvIII siècle, bien que le terme « pomorcy " (en russe : « habitant de la côte ») se trouve attesté plus tôt, depuis le Xvi siècle (Anufriev, 2008, pp. 33-51).

11 Dans le Nord, le terme Pomor'e se rapportait aux XV ${ }^{\mathrm{e}}-\mathrm{xVII}{ }^{\mathrm{e}}$ siècles aux côtes sud-ouest et sud-est de la mer Blanche, les côtes dites Pomorskij (en russe : côtière) et Letnij (en russe : estivale). Plus tard, elles furent utilisées pour désigner des territoires plus vastes du gouvernement d'Arkhangelsk et de la région de Vologda, voire l'ensemble des territoires septentrionaux allant jusqu'à l'Oural. Dans les sources de la fin du $\mathrm{XVII}^{\mathrm{e}}$ siècle, il est utilisé pour désigner les habitants des régions côtières à proprement parler et ceux des bas cours des rivières Onega, Dvina septentrionale et Mezen', qui vivaient de la mer. La formation du groupe culturel des Pomors a duré plusieurs siècles et ne s'est conclue qu'au XvIII ${ }^{\mathrm{e}}$ siècle. Les populations qui ont joué un rôle central dans ce processus sont celles qui avaient quitté Novgorod à une époque tardive, aux XIII $\mathrm{XV}^{\mathrm{e}}$ siècles, avec une importance particulière des migrations en provenance, entre autres, des régions de la haute Volga. L'ethnogenèse des Pomors fait également ressortir des composantes finno-ougriennes (Vlasova, 2005). Il est important de souligner que la ville d'Arkhangelsk a joué dans la consolidation du groupe un rôle inestimable. Elle n'est pas seulement devenue le centre du commerce international de l'État moscovite, mais elle a aussi été le principal foyer économique du Nord européen de la Russie. C'est là que les habitants de la région apportaient le poisson, les produits de la chasse et d'autres marchandises et que se réalisaient non seulement les échanges commerciaux, mais aussi l'intégration culturelle des groupes locaux - c'est là que se formait la conscience commune. Ce n'est donc pas un hasard si les chasseurs et pêcheurs de ces contrées ont commencé à être appelés « Pomors ».

Depuis longtemps, ces hommes pratiquaient des activités liées à la mer, à la chasse, à la pêche, à la navigation et à la construction navale. Depuis la fin du XVII ${ }^{\mathrm{e}} \mathrm{s}$ siècle, on voit grandir parmi eux le nombre des schismatiques, contraints par les persécutions à quitter les régions centrales du pays. C'est en 1695 que s'est formée, à l'ermitage du $\mathrm{Vyg}^{2}$, la toute première des communautés sans-prêtres ${ }^{3}$ - celle des "pomoriens ", ou encore la communauté "côtière ", ou encore des "daniliens", d'après le nom du responsable du Vyg, ou encore du monastère Saint-Daniel, Daniil Vukylič.

Bientôt l'activité économique des Pomors a fait émerger la nécessité de former des responsables maîtrisant l'art de lire et écrire, ce qui a été rendu possible grâce aux contacts permanents des Pomors avec les représentants officiels du pouvoir ainsi qu'avec des étrangers. La lecture et l'écriture se répandirent au XvIII ${ }^{e}$ siècle parmi une partie considérable de la population masculine et même féminine. La présence des vieux-croyants a joué dans ce processus un rôle important, de même qu'elle a exercé une influence non négligeable sur tous les aspects de la vie et de la conscience des Pomors. Même les données incomplètes de la "révision» de 1782 permettent d'apprécier la quantité considérable pour l'époque des personnes alphabétisées en milieu pomor, surtout si on compare cet état à celui des régions agricoles de la Russie. 
Ainsi, dans les volosty de Varzuga et d'Umba, on trouve dans pratiquement chaque famille un ou deux hommes sachant lire et écrire.

Presque chaque Pomor savait par cœur les règles de navigation d'importance locale, leur expérience maritime étant exhaustivement consignée dans le Livre de navigation, un monument de la culture maritime de toute la Russie et même de l'Europe septentrionale. Ce livre rassemblait toute l'expérience collective des Pomors en matière de navigation. Les caractéristiques des activités de chasse et de pêche ont laissé leur trace entre autres sur le caractère de la vie sociale ainsi que sur les pratiques rituelles, que ce soit au niveau familial que des fêtes calendaires, ainsi que dans le partage des tâches au sein de la famille pomore (Bernštam, 1978).

Pomors ont domestique d'immenses espaces dans les mers arctiques, y compris les archipels de la Nouvelle-Zemble et du Spitzberg (en pomor "Grumant»). Leur activité économique, même si elle était basée sur la pêche en mer, incluait néanmoins aussi la chasse aux mammifères marins, la chasse, le commerce (surtout avec la Norvège), l'agriculture, etc., ce qui veut dire que leur économie était vraiment complexe. Suivant les conditions, la part et l'importance de telle ou telle branche pouvaient varier. Pouvaient également varier les terrains de chasse ou de pêche, ainsi que les outils utilisés pour cette dernière. Au cours de la conquête des mers arctiques, les Pomors ont construit une grande variété de types d'embarcations particulièrement adaptées aux conditions du Nord. C'est ainsi que, lorsque dans la deuxième moitié du XIX ${ }^{e}$ siècle, le gouvernement tsariste entreprit d'obliger les Pomors à adopter des types de bateaux plus perfectionnés (de l'avis des autorités), comme des goélettes et d'autres encore, les Pomors lui opposèrent une résistance active en prouvant que leur économie était écologique et efficace (Ružnikov 2005).

Du point de vue linguistique, il n'existait pas un dialecte pomor unique : les chercheurs distinguent tout un groupe de parlers pomors dans le gouvernement d'Arkhangelsk et dans la partie nord de ceux d'olonets et de Vologda (Russkaja 1989). Ils ont été considérés comme des variantes locales de la langue grand-russe et il est naturel que, lors du premier recensement général de la population en 1897, la majorité absolue de la population de l'oblast' d'Arkhangelsk ait répondu qu'elle avait comme langue maternelle le russe (Pervaja, 1905). C'est pourquoi la tentative de présenter les " parlers pomors » comme une langue pomore à part entière suscite des doutes, non du point de vue philologique, mais dans la perspective de "l'autodétermination linguistique » de la population de l'oblast' d'Arkhangelsk, dont témoignent les résultats officiels de tous les recensements. Ceux-ci montrent sans l'ombre d'un doute qu'une écrasante majorité des habitants de cette région reconnaît le russe comme langue maternelle. De plus, on peut difficilement traiter un «parler » comme une « couche» linguistique homogène.

17 Avec les transformations économiques et sociales fondamentales qui caractérisent le XIX ${ }^{e}$ siècle, l'utilisation et le sens du mot Pomor commencent à se perdre. Au $\mathrm{xx}^{\mathrm{e}}$ siècle, et notamment dans sa deuxième moitié, il sort entièrement de l'usage (Bernštam, 1978).

Ainsi, aussi bien les Pomors que les Komis de l'Ǐ̌ma se composent d'éléments ethniques divers et se distinguent très clairement de leurs communautés d'origine. De plus, tous deux se sont formés aux marges de territoires ethniquement bien déterminés et cela s'est reflété dans leur perception d'eux-mêmes. C'est justement la position frontière de ces deux groupes qui permet de les examiner comme des communautés distinctes. 


\section{Les identités}

identité aussi bien pomore que komie de l'Ižma. Mais en quoi consistent ces identités ? Peut-on les considérer comme des identités stables ? Et dans quels groupes d'âge, dans quelles couches sociales cette identité est-elle soutenue le plus activement? Ces questions et les réponses qui leur seront apportées nous permettront de tirer des conclusions quant à l'état de ces groupes culturels aujourd'hui et sur les tendances de leur développement.

Pour ce qui est de la conscience des Komis de l'Ižma, les affirmations de leurs leaders actuels sur le fait que ce groupe forme une communauté ethnique sui generis (Anufrieva 2007) sont sous-tendues par des arguments que nous avons en partie présentés plus haut. Premièrement, ils se distinguent par un haut niveau de solidarité de groupe, ce qui a permis d'arriver à l'établissement d'un document d'enregistrement officiel du groupe, tels le recensement de 2002 et ses résultats. Une initiative du même type, qui avait été entreprise par les Komis d'Udora, avait échoué parce que le groupe avait manqué de solidarité. Deuxièmement, ils ont une identité positive solide. Troisièmement, leur conscience collective présente une nette opposition entre les «Zyriènes » et les « les Komis de l'Ižma », c'est-à-dire que ces derniers ne se contentent pas de se distinguer parmi les Komis, ils s'opposent même aux autres groupes, ce qui s'est manifesté entre autres par un marquage très clair des frontières culturelles grâce à l'opposition entre « Nous » et «Eux » (nous, les Komis de l'Ižma, eux, les Komis) et à l'utilisation de divers types de marqueurs, comme les sobriquets. Parmi ceux-ci, il y en a de péjoratifs et le plus répandu est celui qui appelle les autres Komis « ežvaljad'", même si les linguistes prétendent que la forme originelle devrait être «ežva ljati » (ežva «Vyčegda », c'est-à-dire la rivière le long de laquelle vivent la plupart des groupes komis, ljati « rougeole»). Quatrièmement, les Komis de l'Ižma se distinguent par une mémoire historique particulièrement développée (les personnes d'âge moyen et avancé sont capables en général de citer leurs aïeux jusqu'à la huitième ou neuvième génération). Il faut d'ailleurs noter qu'ils se sont toujours différenciés des autres Komis non seulement par les caractéristiques de leur économie et de leur dialecte, mais aussi par le costume, par les goûts culinaires, par l'esprit d'entreprise et par une orientation plus marquées dans le sens des traditions culturelles russes. Comme l'observent N. Terebihin et D. Nesanelis, «derrière le lustre et l'éclat de leur orthodoxie, les Komis de l'Ǐ̌ma dissimulent leur piété, une tendance innée à la "russitude" et l'aspiration à surpasser les Russes en tout, entre autres dans leur "foi orthodoxe" russe » (Terebihin, 2008, p. 145). Après la création de l'Oblast' autonome komie, les Komis entreprirent de construire leur structure étatique nationale et les Komis de l'Ižma se montrèrent alors opposés à l'enseignement en komi dans les écoles locales, déclarant qu'ils désiraient que l'enseignement fût dispensé en russe. Ils en vinrent même à brûler les abécédaires et les manuels en komi. Il fallut des mesures particulièrement sévères des autorités pour les amener à changer d'avis. Aujourd'hui cette tendance favorable à la «russitude » demeure en partie et ce n'est pas un hasard si certains idéologues du mouvement de l'Ǐma déclarent que les Komis de l'Ižma sont descendants des Novgorodiens, bien que la formation de ce groupe ait de toute évidence associé plusieurs communautés ethniques.

Études finno-ougriennes, 43 | 2011 
21 Qu'est-ce qui nous permet de parler d'un processus de réidentification de groupe ? Nous nous appuyons ici tout d'abord sur les résultats de nos recherches personnelles à la fin des années 1980. Elles ont montré que si la mémoire historique portant sur le passé du groupe était préservée, l'identité locale avait été pratiquement perdue et l'indicateur ethnique principal pour les Komis de l'Ižma, comme pour les autres groupes, était l'ethnonyme komi. Il est vrai que les Komis de la péninsule de Kola et ceux de l'ob ne l'utilisaient guère, considérant qu'il n'était applicable qu'à ceux qui vivaient dans les frontières de la république et se dénommaient «zyriènes » (Kotov, 1996, p. 99). Ce n'est pas surprenant que le processus de réidentification ait été stimulé par l'activité des entrepreneurs ethniques, représentant les groupes locaux. L'identité locale chez les Komis de l'Ižma s'est trouvée engagée, pendant quelques décennies, dans un processus de déconstruction et a laissé la place à une conscience ethnique générale - ce fait est reconnu aujourd'hui par les intéressés eux-mêmes. Les personnes interviewées soulignent en général que dans les années 1960-1980 "nous avions quelque peu oublié que nous étions Komis de l'Ižma» et qu'«à l'époque, on nous instruisait sur le fait que nous étions le même peuple et cela a eu des effets ».

Dans le rajon d'Ižma de la République de Komi, où toute la population, d'après les données du recensement de 2002, était de 21511 personnes (la part des Komis étant de près de $90 \%$ ), 11401 se sont dits Komis de l'Ižma; dans toute la république, ils étaient 12 689. Dans l'Arrondissement autonome nenets, seule une personne s'est dite Komie de l'Ižma, mais des groupes particulièrement importants de Komis de l'Ižma ont été relevés, comme pour pouvait s'y attendre, dans l'oblast' de Murmansk et dans l'arrondissement Jamalo-Nenets. La plus grande partie de ceux qui se disent Komis de l'Ižma sont des ruraux, seuls 1500 urbains ont choisi cet ethnonyme (Šabaev, 2005a).

Ce qui est important, ce n'est pas seulement qu'une partie importante des Komis dont les ancêtres s'appelaient «iz'vatas» ont à nouveau décidé de marquer leur appartenance ethnique par l'ethnonyme local ; il faut également apprécier la solidité des évolutions dans la nature de l'auto-identification et la manière dont l'identité komie de l'Ižma est perçue par les différents groupes d'âge. C'est pourquoi, quatre ans après le recensement, en juin 2008, nous avons fait au village d'Ižma une enquête pilote auprès de la population. Nous avons interrogé uniquement les personnes qui étaient d'accord pour se dire Komis de l'Ižma. L'enquête n'a pas été menée sur la base d'un questionnaire rigoureusement formalisé, mais sous la forme d'entretiens libres avec les personnes sollicitées, au cours desquels nous avons essayé de tirer au clair leur identité ethnique. Nous avons prêté une attention toute particulière aux différences intergénérationnelles dans la perception de l'identité, et de ce fait nous avons identifié trois classes d'âge. Nous avons sollicité vingt-cinq personnes par groupe d'âge (le minimum pour que le groupe soit statistiquement représentatif). Les résultats ont prouvé que dans toutes les classes d'âge, l'idée dominante est que les Komis de l'Ižma forment un groupe particulier de Komis, différents des autres. Un groupe non négligeable considère les Komis de l'Ižma comme un peuple à part entière. Le fait que ces représentations soient particulièrement fortes dans les groupes les plus âgés s'explique par l'importance de la mémoire historique, qu'ils ont préservée. Il est à noter que le soutien à l'idée d'«autodétermination des Komis de l'Ižma » au centre de la région n'a été ni n'est particulièrement significatif. Malgré tout, les résultats obtenus prouvent que la base sociale du mouvement identitaire de l'Ižma est plutôt solide. 
Les formes d'identification des Komis de l'Ižma en fonction de l'appartenance à une classe d'âge

\begin{tabular}{|c|c|c|c|c|}
\hline & \multicolumn{3}{|c|}{$\begin{array}{ll}\text { Par } & \text { tranche } \\
\text { d'âge } & \end{array}$} & \multirow{2}{*}{ Total } \\
\hline & $\begin{array}{l}\leq \\
30\end{array}$ & $31-50$ & $\begin{array}{l}\geq \\
51\end{array}$ & \\
\hline $\begin{array}{l}\text { Les Komis de l'Ižma sont un peuple à part et il ne faut pas les confondre } \\
\text { avec les autres Komis. }\end{array}$ & 2 & 9 & 7 & $\begin{array}{l}18 \\
(23 \%)\end{array}$ \\
\hline $\begin{array}{l}\text { Les Komis de l'Ižma peuvent être considérés comme des Komis, mais ils } \\
\text { se différencient nettement des autres Komis. }\end{array}$ & 24 & 16 & 11 & $\begin{array}{l}51 \\
(65 \%)\end{array}$ \\
\hline $\begin{array}{l}\text { Les Komis de l'Ižma sont des Komis comme les autres et il n'y a } \\
\text { aujourd'hui aucune différence entre eux et les autres Komis. }\end{array}$ & - & 2 & 7 & $\begin{array}{l}9 \\
(12 \%)\end{array}$ \\
\hline Total & 26 & 27 & 25 & $\begin{array}{l}78 \\
(100 \%)\end{array}$ \\
\hline
\end{tabular}

Les conversations ont permis de faire ressortir d'autres particularités intéressantes des sentiments collectifs. En particulier il est apparu que les hommes sont plus disposés à voir les Komis de l'Ižma en tant que communauté à part. Parmi ceux qui ne partagent pas l'idée d'un statut à part, la motivation principale est que l'obtention de celui-ci peut avoir des conséquences négatives : l'augmentation des tensions avec les autres groupes ainsi que la «lumpenisation " croissante d'une partie de la population, réduite en position de parasitisme et de dépendance. Mais de fait les opposants à l'obtention par les Komis de l'Ižma du statut de petit peuple autochtone nient les spécificités du groupe en fonction de raisons purement pragmatiques, et non pas parce qu'ils ne reconnaissent pas la frontière culturelle entre les Komis de l'Ižma et les autres groupes de Komis.

Pour ce qui est du contenu de l'identité pomore, elle aussi est perçue différemment par différents groupes : dans la réalité, il existe aujourd'hui dans la région non point une identité pomore à proprement parler, mais un "champ identitaire » dans lequel entre autres les Pomors sont vus comme une communauté finno-ougrienne à sa manière, comme une communauté slave à part entière ou comme un groupe subethnique (les «Russes pomors »), ou comme représentant une identité non point ethnique, mais locale et régionale. Seule une petite partie des leaders et militants pomors sont prêts à se considérer comme peuple à part entière : la plus grande partie de ceux qui se disent pomors choisit d'autres formes d'appréhension de son identité.

Il est caractéristique que d'après les résultats du recensement de la population de 2002, le plus grand nombre de Pomors était noté dans les villes d'Arkhangelsk et de Severodvinsk (4 000), suivies des rajony de Primor'e et du Mezen'. Le nombre total des Pomors, toujours d'après le recensement, est de 6574 personnes, dont 4779 d'urbains et 1792 de ruraux (Itogi, 2004, p. 14).

27 À la différence du rajon de l'Ižma de la république komie et de la presqu'île de Kola, où la population a été vivement encouragée à s'inscrire comme étant « Komis de l'Ižma », 
l'oblast' d'Arkhangelsk n'a pas connu de campagne comparable, bien qu'il y ait eu quelques publications dans la presse locale sur la renaissance d'une identité pomore. Les agents du recensement n'ont pas rencontré tout le monde personnellement, parfois l'appartenance ethnique a été notée sur la base d'indications données par la famille ou par des amis, et il est également arrivé que les agents n'aient gardé que la première partie du groupe "Russes pomors", dans la mesure où les documents explicatifs du recensement ne prévoient pas le cas de double appartenance ethnique. De nombreuses personnes, comme le font remarquer les leaders du mouvement pomor, n'ont pris conscience qu'après le recensement du fait qu'ils auraient pu se dire pomors.

Il n'en reste pas moins que le résultat obtenu reste proche des dispositions d'esprit réelles dans l'oblast' d'Arkhangelsk et dans quelques autres régions que l'on peut rattacher à la Pomorie historique (Bulatov, 2003). Une partie importante des personnes originaires de l'oblast' d'Arkhangelsk et dont les aïeux se dénommaient pomors, disent d'eux-mêmes consciemment qu'ils sont russes, bien que souvent ils affichent une attitude positive envers l'idée pomore. Qui plus est, dans les villages pomors des berges de la mer Blanche, où la population reste encore aujourd'hui liée à la pêche au poisson et à la chasse aux mammifères marins, les habitants s'appellent Pomors, alors qu'ils ne définissent pas ce concept de la même manière que le fait l'idéologie du mouvement pomor.

Il est curieux de noter que dans le passé la population des vieux-croyants de l'oblast' d'Arkhangelsk s'est aussi identifiée de manières diverses. Les habitants des volosty du Sud ne faisaient pas partie des Pomors et s'appelaient "Vagan " (du nom de la rivière Vaga). Mais même dans les volosty du Nord, tout le monde ne se dénommait pas pomor. Une partie de ces populations disent encore aujourd'hui : "Nous ne sommes pas des Pomors, nous sommes des Novgorodiens ", évoquant les ancêtres qui étaient venus jadis de Novgorod s'installer dans ces régions. Si on leur demande quand leurs ancêtres sont allés s'installer dans le Nord, ils répondent en général : « Il y a trois cents ans. » De plus, dans l'oblast' d'Arkhangelsk, les habitants d'un certain nombre de villages continuent de se dénommer "Tchoudes». D'après le témoignage de bien des chercheurs, le mot tchoude était utilisé comme marqueur local pour désigner des personnes, une partie de la population ou la population de certains villages, et il rendait compte d'une origine fennique (Kirpičnaja, 1991). Des endroits de ce type, il s'en trouvait beaucoup dans le nord et surtout dans l'oblast' d'Arkhangelsk. Mais il est important de noter ici que tous ces marqueurs sont porteurs d'une connotation, soit positive soit neutre. Le terme peuple autochtone (КОРЕННОЙ НАРОД) est plutôt ambigu, et il apparait comme assez proche du terme aborigène (ТУЗЕМЕЦ).

Par exemple, dans le rajon du Mezen', qui fait partie de l'okrug nenets et où depuis des temps immémoriaux les Pomors sont en contact avec les Nenets, la définition des Pomors comme d'un « petit peuple autochtone » (КОРЕННОЙ МАЛОчИСЛЕННЫЙ НАРОД) est interprétée par la population locale comme une tentative de les assimiler aux Nenets, ce qui pour la conscience collective de la population locale est inacceptable, dans la mesure où cette population, dans la hiérarchie sociale, s'est établie depuis longtemps bien plus haut que les Nenets. Ceux-ci, en effet, n'étaient pas vus comme un peuple chrétien (les Komis de l'Ǐma avaient la même attitude à l'égard des Khantys dans le bas cours de l'Ob). Ainsi, c'est la population vivant de chasse et de pêche, et qui a préservé un mode de vie historique, qui est la moins prête à se définir comme communauté spécifique du point de vue ethnique (bien que les habitants des villages 
pomors ne soient pas en mesure de répondre de manière déterminée à la question " qui sont les Pomors? ») et distincte de la communauté ethnique dominante dans le pays. D'ailleurs, des activités militantes résolues parmi les habitants des villages côtiers pourront changer les attitudes de la population, alors qu'une oppression plus accentuée de la part des institutions d'État, comme un renforcement de la garde-frontière, des organismes de protection du poisson et autres, pourra amener la population à rechercher de nouvelles formes d'adaptation aux conditions d'existence en mutation. Ici, l'ethnicité pourrait devenir le moyen de préserver un mode de vie tout en répondant de manière satisfaisante aux intérêts économiques de la population pomor de la mer Blanche.

31 Il est compliqué de systématiser les données sur l'identité pomore, dans la mesure où elle s'est formée historiquement sur un arrière-plan d'autres identités ethniques et régionales, qu'elle n'a longtemps pas été actualisée et qu'encore aujourd'hui, elle est insuffisamment déterminée et fortement mythologisée. Elle se fonde sur un lien mythique entre les Pomors et la mer, le territoire au bord de la mer et la conscience de ce que ce territoire n'est pas une périphérie, mais une frontière. La Pomorie, ce n'est seulement la frontière nord de la Russie, son bastion dans le nord, c'est aussi une frontière culturelle, un avant poste sui generis de la culture russe.

32 Faire de l'idée pomore et de l'identité pomor une partie intégrante de l'idéologie régionale, comme le proposent les leaders pomors, ne sera possible qu'en promouvant un discours intellectuel sur les idées de Nord russe et de Pomorie, et dans la conscience de masse l'idée de «Pomor» et d'« habitant du Nord» (Северян). Pour l'instant, les idéologues du mouvement pomor eux-mêmes, sans le faire exprès, provoquent un conflit d'identités à Arkhangelsk, car ils opposent les Pomors aux Sibériens (Moseev, 2005), ce qui en partie est dû à la tradition culturelle d'après laquelle il était convenu de distinguer les «étrangers» (ЧУЖАНОВ) (c'est-à-dire les personnes venant d'ailleurs) des Pomors, et, dans la période soviétique, de classer la population (dont la composition a fondamentalement changé) en "locaux » et "recrutés », c'est-à-dire ceux qui vont vivre dans le Nord sur la base de contrats de travail.

33 Il est évident que l'attention portée aux identités locales, leur reproduction renforcée, ont leur logique et ont provoquées par les mutations, les processus sociaux et politiques qui ont lieu en Russie et dans le monde contemporain en général. Les chercheurs travaillant sur les processus de formation des identités contemporaines observent: «Dans les sociétés contemporaines, dans lesquelles les individus doivent faire face à une multitude d'attentes concernant leurs rôles sociaux, cette situation implique la formation d'identités multiples. Suivant le contexte, la détermination d'identités partielles acquiert du sens ou passe au second plan, ce qu'il faut comprendre non seulement comme une réaction passive à l'environnement ou aux exigences du groupe, mais aussi comme une distribution individuelle raisonnée des priorités » (Voronkov, 1998, p. 13).

34 La logique de la refonte de l'identification à des groupes sociaux se comprend d'autant mieux si on tient compte d'un autre phénomène non moins important, en rapport avec les réformes radicales de la société et de ses structures de base, à savoir le traumatisme culturel, vécu jusqu'à aujourd'hui, en résultat de l'éclatement de l'ancien système de valeurs, dans la société russe et ses conséquences. De plus, le processus de refonte des anciennes identités est étroitement lié à l'établissement des nouvelles identités, puisque dans le corps social en mutation dynamique, les anciennes identités 
disparaissent ou s'affaiblissent et inévitablement, de nouvelles apparaissent. Comme le font remarquer E. N. Danilova et V. A. Jadov, dans un tel corps social

il est en principe impossible d'avoir une identité sociale stable. Et alors ce que nous appelons une «identité de crise » émerge en tant qu'état ordinaire des individus, qui sont contraints, par le contexte objectif des transformations sociales permanentes, de suivre ou de refléter leurs orientations dans l'espace « Nous-Eux » - dans leur auto-identification et dans leur statut social (Danilova, 2004, p. 30).

De nouvelles identités se forment, car

dans la construction de nouvelles identités, les mécanismes psychologiques de rapprochement et d'éloignement des distances culturelles jouent un rôle important, quoique souvent dissimulé. Avec leur aide, et à l'aide de leviers soigneusement calibrés, la conscience individuelle et de groupe semble chercher et déterminer sa place dans le monde en mutation (Lebedeva, 1997, p. 81).

Ces positions des chercheurs russes correspondent à celles élaborées par Sztomka et par Vogt (Sztomka, 2004 et Vogt, 2005). Le fond de leur approche de l'analyse des mutations sociales tient à ce que le processus de mutations culturelles est étroitement corrélé aux transformations politiques et économiques. Les sociétés stables sont caractérisées par un équilibre culturel qui se traduit par des traditions, des valeurs, des identités. D'importantes transformations sociales bouleversent cet équilibre et conduisent à l'apparition de nouveaux symboles, valeurs et identités, qui coexistent avec les anciens. D'ailleurs souvent intervient la déception par rapport aux valeurs contemporaines, ce qui conduit à un attrait particulier envers les expériences et les valeurs du passé.

Les mutations économiques et sociales dans la Russie postsoviétique ont été considérables et elles ont conduit, ce qui n'est pas surprenant, à une actualisation brusque de l'ethnicité, phénomène qu'on a appelé ethnicité révoltée, révolution ethnique, ou encore paradoxe ethnique du monde moderne.

Le passage à une économie de marché a conduit non seulement à une stratification sociale accentuée parmi les citoyens, mais aussi à une répartition des régions russes entre régions donneuses et régions en dépression (régions subventionnées), et, à l'intérieur même des régions, à une différenciation d'après les indicateurs de réussite économique et sociale. L'oblast' d'Arkhangelsk, d'après le niveau de vie de ses habitants et de nombreux autres indicateurs économiques, a beaucoup de retard par rapport aux régions-leaders, alors que les rajony que l'on peut appeler "traditionnellement maritimes" (les rajony de Primor'e et du Mezen') doivent être classés parmi les territoires en stagnation. Le rajon d'Ižma de la République komie pour sa part fait aussi partie des zones en dépression, où il existe une situation sociale très compliquée (haut niveau de chômage, de suicide, etc.). Ne possédant intrinsèquement rien qui stimule le développement, les rajony en question vont chercher des stimulants dans leurs ressources culturelles.

\section{Les institutions sociopolitiques et leur idéologie}

La mobilisation politique de l'ethnicité, qui a commencé à la fin des années 1980 et au début des années 1990, a conduit non seulement à un accroissement du niveau d'activité politique des communautés ethniques et à l'actualisation de la conscience ethnique générale, mais a aussi permis la renaissance des consciences identitaires 
locales, la formation d'organisations ethnopolitiques fondées sur la mémoire historique des groupes ethnographiques du passé.

En 1990, avec le soutien des autorités locales, le $1^{\text {er }}$ congrès constitutif tenu au village d'Ǐžma a fondé le mouvement des Komis de l'Ižma «Iz'vatas », association reconnue au niveau de la République. Plus tard, des sections ont été créées dans la péninsule de Kola (au village de Lovozero), et dans les okrugs autonomes nenets et iamalo-nenets. Au premier congrès, Vitalij Kanev, qui sera d'ailleurs par la suite élu président de l'organisation, a présenté un rapport intitulé « Préserver le groupe ethnique des Komis de l'Ižma». Dans ce rapport, ce groupe est présenté comme une «ethnie » (ЭТНОС) formée par un mélange de Russes, de Komis et de Nenets des forêts, qui se distingue fondamentalement, du point de vue de la langue, de la culture et du mode de vie, « des autres populations komies ».

41 Depuis la fondation du mouvement des Komis de l'Ižma sur la base d'idées qu'il défend, on a constaté une dualité dans la conception de la communauté ethnique proprement dite. D'une part, les idéologues du mouvement affirment que « Les Komis de l'Ižma font partie intégrante du peuple komi », et de l'autre, depuis le tout début, il est question d'une "ethnie» de l'Ižma, dont la formation remonte à la deuxième moitié du $\mathrm{XVI}^{\mathrm{e}}$ siècle (Hatanzejskij 2000). Il est à noter que le terme ethnie, concept clé dans l'ethnographie soviétique (Tiškov 2003) a été activement repris par les « entrepreneurs de l'ethnicité ", tout en étant interprété à leur manière, c'est-à-dire de manière différente de l'acception scientifique traditionnelle. Il n'en reste pas moins que le fait de marquer les Komis de l'Ižma comme une " ethnie », leur a permis - et les militants l'ont bien compris - d'élever le statut culturel de leur groupe.

Pour commencer, ils s'étaient fixé des tâches clairement définies, mais manquant quelque peu de précision:

- défendre et mettre en œuvre les droits souverains et des intérêts des Komis de l'Ižma à tous les niveaux de la gestion;

- préserver et développer la langue et l'originalité culturelle des Komis de l'Ižma ;

- contribuer à la renaissance de la chasse et de la pêche, faire connaître dans la population l'histoire de la région de l'Ižma et les traditions de ses habitants, organiser les loisirs des gens... (Hatanzejskij, 2000, p. 3)

De plus, l'un des éléments importants de l'idéologie était le concept du « siècle d'or des Komis de l'Ižma ", que représente le tournant des XIXe et Xx siècles. Les Komis de l'Ižma se distinguaient alors des autres groupes komis par leur richesse et par leur esprit d'entreprise. Le florissement du groupe était étroitement lié à l'élevage du renne. C'est de là qu'est née l'idée de la création des conditions optimales pour le développement de cette branche, et l'idée d'attribuer au rajon d'Ižma le statut de " rajon national » (Filippov, 1991).

Le deuxième marqueur identitaire dans les constructions idéologiques du mouvement a été la figure culturellement originale de l'Iz'vatas.

Il se distingue des autres Komis par l'originalité de sa langue, de sa culture, par la richesse de ses traditions. Dans son développement historique, les Komis de l'Ižma, que l'on appelle Iz'vatas ont pu survivre grâce à leur naturel travailleur, à leur sagesse et au respect envers les autres peuples. Les traits caractéristiques des Komis de l'Ižma sont la modestie et la patience, le laconisme et la timidité. L'écrivain Maksimov a noté la présence dans leur caractère de capacité d'initiative, de 
rationalité, d'inventivité et d'adresse - bref tout ce qui caractérise des individus portés vers le commerce.

d'exploitation traditionnelle des ressources, la quête de stimulants pour le développement $\mathrm{du}$ groupe grâce à de nouvelles relations avec les exploitants des ressources naturelles et si, avant, le mouvement avait essayé d'achever ces objectifs en modifiant le statut du rajon, il était progressivement parvenu à la conclusion qu'il était raisonnable d'essayer d'obtenir un changement dans le statut du groupe lui-même. Déjà à cette étape, les évolutions dans les positions du mouvement de l'Ižma n'étaient pas toujours en accord avec les positions du mouvement komi général (Коті vojtyr, Коми войтыр). Mais dans son ensemble, l'idéologie d'« Iz'vatas » ne se présente pas sous une forme conceptuelle achevée, et apparaît comme relativement indéterminée, alors que ses leaders n'accordent que peu d'attention à l'élaboration et à la propagation dans la population de concepts idéologiques; apparemment, ils préfèrent supposer intuitivement que la mémoire historique des Komis de l'Ižma est plutôt forte et pèse fortement sur les dispositions d'esprit collectives.

Dans le positionnement politique de l'association « Iz'vatas », un jalon important a été la préparation et le déroulement du recensement de 2002. En août 2002, la section de l'association «Iz'vatas » du village de Lovozero (oblast' de Murmansk) a adopté un appel à leurs homologues en pays komi, en leur demandant de définir leur appartenance ethnique au cours du recensement non point comme Komis, mais comme Komis de l'Ižma. En septembre de la même année, le Conseil du mouvement a déclaré pratiquement à l'unanimité son soutien à cet appel. C'est alors que les militants de l'Ižma et les conseillers régionaux du « rajon d'Ǐžma sont intervenus avec un appel à inclure les Komis de l'Ižma dans la liste des peuples autochtones qui a été confirmée officiellement par le gouvernement de la Fédération de Russie en 2000.

Le 28 juin 2003, le cinquième congrès du mouvement « Iz'vatas » a eu lieu à Ižma. Y ont assisté le premier responsable de la république komie Vladimir Torlopov et le ministre de la Culture et des Questions nationales, Marija Kuz'boževa. Cette dernière, dans son intervention, a mis l'accent sur l'unité du peuple komi, qui repose sur la communauté des sorts historiques et sur la proximité culturelle de tous les Komis. Le Congrès a néanmoins adopté la décision d'obtenir pour les Komis de l'Ižma le statut de «petit 
peuple autochtone ${ }^{4} »$. Les raisons de cette aspiration sont surtout économiques. Le nouveau président du mouvement explique la décision prise de la sorte :

Malheureusement, jusqu'ici, les droits et les avantages de nature socio-économique ainsi que les avantages fiscaux dans le domaine de l'exploitation des ressources naturelles, dont les Khantys et les Sames recueillent déjà les fruits, ne s'étendent pas aux Komis de l'Ižma. Par exemple, les groupes ethniques assimilés aux petits peuples autochtones du Nord ont droit à un contrôle médical annuel, et depuis 2004 ils ont le droit de faire un service alternatif au lieu du service militaire. De plus, les représentants des petits peuples sont dispensés de taxes sur l'utilisation des ressources forestières pour leurs propres besoins. Sur les terres reconnues officiellement comme zone d'exploitation traditionnelle par les petits peuples, toute exploitation industrielle polluante pour l'environnement est interdite. Pourquoi l'un des peuples qui sont nos voisins peut-il bénéficier de ces droits, et les autres non? Pourtant telle est aujourd'hui la situation... La dénomination de petit peuple autochtone doit bien sûr s'appliquer non seulement aux éleveurs de rennes, mais aux habitants des villages du rajon de l'Ižma, et également aux Komis de l'Ižma habitant dans d'autres régions. (Sivkova, 2003)

L'intervention de la responsable d'« Iz'vatas » permet de déduire qu'en pays komi, de même que dans le Nord européen en général, un conflit a émergé à propos des statuts culturels. Ce n'est pas un hasard si l'initiative de faire des Komis de l'Ižma un groupe à part est partie de la péninsule de Kola. Là, la différence de statut entre les Komis et les Sames, qui vivent dans les mêmes agglomérations, est ressentie de manière particulièrement douloureuse. C'est à partir de ce moment-là que les relations se sont tendues entre l'association «Iz'vatas » et les leaders du mouvement komi.

51 L'insistance sur les intérêts spécifiques des Komis de l'Ižma et sur leur statut à part, incontestablement, a conduit à leur éloignement par rapport à la communauté ethnique à laquelle ils se rapportaient et au mouvement komi en général. Fin 2004, les Komis de l'Ižma ont adhéré à l'Association des petits peuples autochtones du Nord, de Sibérie et d'Extrême-Orient (RAIPON) et en avril 2005 leur délégation a participé au cinquième congrès de cette organisation. Cependant, les disputes autour de l'objectif déclaré des Komis de l'Ižma de se proclamer petit peuple du Nord ne sont pas retombées.

Dans l'un des entretiens accordés par l'ancien ministre de la Culture et des questions nationales M. Kuz'boževa (une militante du mouvement komi), par exemple, on trouve :

Les Komis de l'Ižma prétendent qu'ils ne sont pas komis. Ce faisant, ils renient toute l'histoire du peuple komi. Ce faisant, ils se séparent, ils s'isolent des autres Komis, lesquels, ayant leurs caractéristiques ethniques et linguistiques, font pourtant partie d'un peuple qui est autochtone, même s'il n'est pas petit - les Komis, les Komis-Zyriènes. L'intention d'obtenir un statut à part, afin de passer sous la protection de l'État, est humiliante pour le peuple komi. (Mezak, 2004)

Début avril 2005, les leaders du mouvement des Komis de l'Ižma se sont vus adresser une lettre officielle qui les mettait en demeure de prouver scientifiquement l'existence d'une ethnie komie de l'Ižma (Smetanin, 2005), ce qui a suscité une certaine agitation parmi les membres d'« Iz'vatas ». Cette position signifiait que les autorités locales aussi bien que les leaders du mouvement komi ne partageaient pas l'idée de liberté culturelle formulée dans le rapport préparé par le Programme de développement de l'Organisation des Nations Unies "La liberté culturelle dans le monde multiforme contemporain ", que V. A. Tiškov appelait à suivre (Tiškov, 2005). De plus, la situation créée autour des Komis de l'Ižma témoigne d'un conflit de plus en plus apparent, le conflit des identités. 

a changé fondamentalement. Les premiers à s'être montrés intéressés aux revendications des Komis de l'Ižma ont été les législateurs, et il a été question d'en discuter au Conseil d'État de la République komie. Par la suite, le chef de l'État, Vladimir Torlopov, a fait une déclaration particulièrement importante, portant sur la demande des Komis de l'Ižma d'être inclus dans la liste des petits peuples autochtones. Le $1^{\text {er }}$ juillet 2008, à une conférence de presse, il a déclaré en réponse à la question d'un journaliste :

Je ne vois rien de terrible à ce qu'on leur donne satisfaction. Je suis convaincu que ceci ne conduira pas à un schisme dans le mouvement finno-ougrien. Il est question de certaines préférences dans la sphère socio-économique, destinées à améliorer les conditions de vie des gens dans certaines conditions précises. Personne ne poursuit sur cette question quelques intérêts politiques que ce soit. Les gens veulent vivre bien, travailler dans les branches traditionnelles - culture de la terre, chasse, pêche - préserver leur mode de vie même après l'arrivée dans leur région de la civilisation contemporaine. Pourquoi ne pas les soutenir dans cette entreprise?

D'après le gouverneur, la position d'une partie considérable de la direction de « Komi Vojtyr » ne doit pas empêcher que les Komis de l'Ižma s'adressent aux instances supérieures (Vorvraščenie, 2008).

En même temps, Valerij Markov, leader du mouvement komi et président du Comité consultatif des peuples finno-ougriens, à l'inverse, s'est exprimé en termes très critiques à ce sujet, et a déclaré que cette démarche aboutirait à détruire l'unité du peuple komi, car il s'agit d'une décision qui peut déclencher des réactions en chaîne et amener d'autres groupes komis - ceux d'Udora, les Komis du sud - à désirer eux aussi le statut de peuple autochtone (Sivkova, 2008). Au Ve Congrès mondial des peuples finnoougriens (juin 2008, Hanty-Mansijsk), la section des droits et de la politique ethnique a recommandé d'inclure l'appel de l'association «Iz'vatas » demandant le statut de petit peuple du Nord dans la résolution du Congrès (cette demande avait été adressée par la présidente de l'association V. Anufrieva en mai 2008 au président de la Fédération de Russie D. Medvedev et au Premier ministre V. Putin). Mais, après discussion, ce point n'a pas été inclus dans la résolution.

Le processus de fragmentation des communautés ethniques et de renaissance d'anciens ethnonymes n'a pas touché seulement les Komis. Chez les Oudmourtes, par exemple, les Bessermans se sont affirmés, chez les Mordves, les Erzyas et les Mokchas, chez les Tatars, les Krjašen, les Mišars et les Tatars de Sibérie, et chez les Russes - les Cosaques, les Kamtchadales et les Pomors. étape de développement de l'idée pomore.

À notre avis, il faut aller chercher à l'origine des tentatives de construire une nouvelle identité pomore, entre autres raisons, les intérêts politiques des élites locales. C'est l'élite régionale d'Arkhangelsk qui avait avancé l'idée de faire de cette ville la capitale du nord de la Russie et de rassembler autour d'elle toutes les régions du Nord. C'est pourquoi, au début des années 1990, l'idée pomore commença à émerger, en tant que fondement d'un mouvement sociopolitique régional, opposé au centre fédéral. C'est alors aussi qu'avait émergé l'idée d'une République pomore (alors qu'à la même époque on parlait activement d'autres républiques - République de l'Oural, République de Vologda, et bien d'autres). C'est pour la mise en œuvre de cette idée qu'en 1992 toute une série de partis politiques et d'organisations de l'oblast' d'Arkhangelsk avait 
enregistré un Centre culturel national appelé "Renaissance pomore ». Dès l'étape initiale, ce mouvement proposait de consolider politiquement les régions du Nord européen de la Russie. Mais le centre fédéral n'avait pas l'intention de rassembler les régions du Nord, toutes militarisées, en une République unique : il perçut dans l'idée de renaissance pomore l'amorce d'une sorte de "séparatisme pomor", qu'il combattit activement. De nombreux leaders du mouvement, même s'ils intervenaient en faveur d'une république, refusèrent cependant à l'époque de fonder un mouvement pomor ethnopolitique. En 1994, le mouvement politique "Renaissance pomore» éclata en différents groupements. Les tentatives ultérieures d'unifier les organisations pomores dans le cadre d'un "Monde pomor» ne furent pas couronnées de succès. Il n'en reste pas moins que «l'idée pomore» s'est fortement implantée dans le discours politique régional.

En 1998, le leader du mouvement "Renaissance démocratique du Nord» (qui a cessé d'exister en 2001), Aleksandr Ivanov, essaya d'en exprimer l'essence :

Les Pomors ont un sens particulier de leur valeur et de l'amour de la liberté. La raison en est que le Nord n'a pas connu le droit féodal: la forme principale d'organisation de la vie sociale n'était pas la communauté (овщинА), mais l'artel. Les Pomors n'ont pas le sens de l'ennemi, dans la mesure où les ressources naturelles ont toujours suffi pour tout le monde, et que l'étranger était perçu non point comme un concurrent, mais comme un partenaire commercial. Depuis les plus anciens contacts avec l'Europe (par la mer, Édimbourg, Oslo et Brême sont plus proches que Moscou), ils ont élaboré une conscience tournée vers l'Europe occidentale, ignorant la xénophobie, respectueuse des institutions démocratiques. Les Pomors sont historiquement caractérisés par un certain mépris à l'égard du pouvoir moscovite - qu'il soit tsariste, soviétique ou postsoviétique - pour son caractère mensonger et cruel, ainsi qu'à l'égard de l'arbitraire qu'il produit. Les Pomors essayent de travailler non point pour l'État, et essayent d'en dépendre le moins possible. Bien avant la révolution, la population du Nord se distinguait par son haut niveau d'alphabétisation. Pour les hommes du Nord, l'éducation, la culture et la recherche ont une valeur inestimable. Ce qu'on appelle, en Russie centrale et méridionale, patriotisme - la haine de l'Ouest, de la liberté et de la démocratie, de l'intelligentsia - de notre point de vue de Pomors n'est pas du patriotisme, mais de la muflerie. La rude nature du Nord a conditionné les traits de caractère des Pomors : humilité, patience, fermeté, un mélange sui generis d'esprit pratique et de mysticisme. Confrontés au déchaînement des forces de la nature, nous demandons à Dieu miséricorde, et confrontés à la tyrannie moscovite nous demandons aussi miséricorde à Dieu - la demander aux tyrans n'a aucun sens... (Filatov, 2002, p. 65)

61 Les mythes historiques des libres Pomors et des profondes traditions démocratiques que le pouvoir moscovite a anéanties sont faciles à mettre en cause, dans la mesure où les immenses terres pomors étaient, dès le $\mathrm{xv}^{\mathrm{e}}$ siècle, gérées par les boyards et que toute la vie de la population était strictement règlementée. Il n'en reste pas moins que ces mythes restent fondamentaux pour le mouvement pomor, de même que les mythes de leur ethnogenèse, c'est pourquoi ils ne cessent de se reproduire.

En soulignant l'importance des mythes ethniques dans l'idéologie, V. Šnirelman montre que

le mythe joue un rôle instrumental - il répond à une tâche contemporaine tout à fait concrète, qu'il s'agisse de revendications territoriales, d'exigences d'autonomie politique ou d'aspiration à contrecarrer le nivellement culturel et à préserver son héritage culturel. (Šnirelman, 2000, p. 14) 
63 ensemble de mythes concerne les caractéristiques du caractère pomor, de la mentalité pomor. Celle-ci se trouve explicitée de la manière la plus achevée dans l'ouvrage L'écologie pomore :

Être pomor, c'est un style de vie, un algorithme de comportement dans un environnement donné. Pour le Pomor, « la mer est notre champ ». C'est pourquoi il est curieux d'apprendre que l'ancien gouverneur de l'oblast' d'Arkhangelsk s'est déclaré pomor au dernier recensement. Est-ce qu'on peut être ancien Pomor, ou d'origine pomor? Non! On peut être descendant de Pomors. Un Pomor, ce n'est pas seulement quelqu'un qui vit au bord de la mer, c'est avant tout quelqu'un qui suit des traditions précises, rigides, en partie autolimitatives de comportement dans son environnement naturel et dans la sphère sociale. L'objectif de ce comportement est d'atteindre à l'équilibre naturel, à l'harmonie des relations dans le système « individu (famille, clan) - nature ». Atteindre cet équilibre permet de garantir une existence longue et stable dans les conditions rudes de la nature septentrionale. (Lisničenko, 2007, p. 81)

L'arrivée au pouvoir du gouverneur Anatolij Efremov, pomor d'origine, comme le suggèrent les experts de la région, non seulement n'a pas aidé le mouvement pomor, mais a freiné son développement. Efremov s'est positionné comme "le Pomor numéro un ", le «Pomor russe » et il ne supportait pas l'existence d'autres leaders. Nikolaj Kiselev, le successeur d'Efremov au poste de gouverneur de l'oblast' d'Arkhangelsk, était opposé à l'idée de " renaissance pomore », même si en 1989 il avait créé le mouvement "Les Pomors », qui avait ressemblé surtout des fonctionnaires et des administrateurs et qui avait pu obtenir quelques sièges aux élections locales. En même temps, il ne mit guère d'obstacle au développement du mouvement pomor et son administration apporta même son soutien à la demande des leaders pomors d'obtenir le soutien du gouvernement. Le nouveau gouverneur Il'ja Mihalčuk est loin de la problématique pomore et ne soutient ni le mouvement pomor ni les idées pomores.

Le développement du mouvement pomor a été encouragé par les résultats du recensement de 2002, qui ont montré que l'identité pomore existait réellement et pouvait être fixée dans les statistiques.

C'est en 2003, à Arkhangelsk, qu'a été enregistrée l'autonomie ethnique et culturelle des Pomors de la ville, et début 2004, une communauté pomore a été fondée en tant que communauté relevant des petits peuples du Nord. Les leaders actuels des Pomors déclarent qu'ils ne sont ni un groupe ethnographique ni une sous-ethnie du peuple russe, mais une «ethnie » à part entière. Ils ajoutent que cette ethnie est finnoougrienne, puisque son substrat est constitué des tribus finno-ougriennes qui vivaient dans le nord de l'Europe avant l'arrivée des colonisateurs russes. À notre avis, la renaissance des Pomors et la construction d'un nouveau contenu à leur identité s'expliquent en grande partie par des intérêts politiques et économiques, et non pas par l'aspiration à l'isolement culturel et à la préservation de leurs traditions.

67 I. Moseev, président du Centre culturel national d'Arkhangelsk, a formulé ainsi, dans la conclusion à son rapport L'autodétermination ethnique et l'ethnogenèse des Pomors, le fondement idéologique de l'autonomie ethnique et culturelle des Pomors :

Malgré d'innombrables tentatives d'assimiler les Pomors et de les présenter uniquement comme une partie intégrante de la puissante ethnie grand-russe (groupe ethnographique, sous-ethnie, population, groupe social, etc.), les Pomors ont préservé leur conscience ethnique, ce dont témoignent les résultats du recensement de 2002. Les Pomors sont une ethnie à part, dont la culture originelle n'est pas importée de l'extérieur (c'est-à-dire de Russie), mais a émergé au cours de 
la fusion progressive des cultures finno-ougriennes "pré-pomores » locales et des cultures des premières anciennes populations russes (mais non " grand-russes "!). À la différence des autres communautés russes (par exemple les gens de Viatka, les Krivitches et autres), qui se sont fondues dans l'ethnie grand-russe perdant leur conscience ethnique, les Pomors l'ont conservée jusqu'à aujourd'hui et continuent de se considérer pomors. Aujourd'hui, le terme pomor distingue activement une population autochtone du Nord russe de la communauté superethnique des "gens du Nord ", la population non autochtone de la région. (Šabaev, 2003)

Cette position est partagée également par le président de l'autonomie ethnique et culturelle de la ville d'Arkhangelsk, Pavel Esipov, qui note :

Les ancêtres des Pomors étaient des tribus finno-ougriennes, comme les Sames, les Caréliens (d'où l'appellation de «Petite Carélie»), les Häme (d'où le nom «Emeck»). Plus tard sont venus s'installer sur les terres qu'ils habitaient les premiers peuples slaves, chacun ayant son appellation propre. (Esipov, 2006)

européen. Entre autres, I. V. Vlasova note :

Les Novgorodiens et les Rostoviens, qui forment la base de la population russe du Nord, même s'ils représentaient des communautés régionales liées à la terre, relevaient d'une même ethnie, alors qu'ils avaient des origines mêlées puisqu'ils avaient vécu et s'étaient développés dans différentes conditions naturelles et économiques et en allant s'installer en Europe orientale, entre autres dans le Nord, ils avaient trouvé sur leur passage différents groupes d'origine finno-ougrienne. C'est ainsi que les groupes locaux de la population russe ont formé et préservé longtemps des traits particuliers, manifestes dans leur culture populaire. (Vlasova, 2005, p. 39)

70 Bien sûr, l'histoire ethnique et la mémoire historique des gens ne dépendent pas rigidement l'une de l'autre, car la mémoire s'appuie souvent non point sur des faits réels, mais sur les mythes et les stéréotypes culturels dont il a été question plus haut. Et la signification des mythes et des stéréotypes est bien supérieure à celle des idées et des connaissances rationnelles.

71 Il est également important de noter ici que la classification scientifique russe qui sert de fondement à la politique nationale, renforce clairement l'opposition stéréotypée "Russes» - "autres", ce qui en même temps simplifie et rend indéterminé le deuxième terme de l'opposition. À ce sujet, S. Sokolovskij note :

Cette expression indéterminée elle-même, les autres, qui permet à chaque instant d'inclure dans cette catégorie des éléments nouveaux, témoigne du caractère variable de la russitude. (Sokolovskij 2001)

72 C'est justement le caractère indéterminé et pluriel de la perception des communautés ethniques qui apparaît dans la tradition politique et scientifique russe (suscitée par la hiérarchie ethnique institutionnalisée) qui ouvre de larges possibilités pour la construction de communautés ethniques et de groupes culturels. Dans cette perspective, une importance extrême doit être accordée au terme pomor, qui s'est enraciné dans la conscience de l'élite intellectuelle en tant que "marque » locale profondément ancrée.

73 Aujourd'hui, le « marqueur » pomor est activement utilisé aussi bien par les leaders du mouvement pomor que par le personnel politique local et par les intellectuels dans des buts profondément pragmatiques, alors même que dans les communautés locales l'utilisation de cette "marque » fait l'objet d'un consensus particulier, car sa valeur positive est soulignée par les représentants de différents groupes sociaux. Ce n'est pas 
par hasard que nous faisons appel au terme marqueur : en effet, dans leurs efforts pour déterminer le sens de «l'idée pomore» pour la communauté locale, bien des intellectuels d'Arkhangelsk l'ont utilisée indépendamment les uns des autres au cours d'interviews. Ceci est révélateur dans la mesure où les pratiques discursives évoquées se réfèrent à un moyen d'établir des frontières culturelles, à leur caractère construit. Il faut pourtant souligner que cette frontière culturelle construite a été socialement approuvée.

En même temps, l'attitude envers les idées pomores est plutôt contradictoire aussi bien dans la société que dans l'élite politique de la région. D'une part, à l'époque des campagnes électorales, le marquage pomor, Pomor, le pays pomor, est invariablement utilisé dans les documents de propagande et est extrêmement important pour le personnel politique, dans la mesure où d'une part il souligne leur attachement à la région et aux intérêts locaux, alors que d'autre part les leaders du mouvement pomor sont souvent accusés de séparatisme, de former un «lobby norvégien » et de recevoir de l'argent de la Norvège pour mener leurs actions.

En même temps, cette double perception n'a pas empêché le mouvement pomor de se développer de manière fort active et de devenir un acteur politique marquant dans la région. Une étape importante dans l'affirmation de ce mouvement en tant qu'organisation ethnopolitique a été le Congrès du peuple pomor, tenu à Arkhangelsk en septembre 2007. Ce Congrès, à la différence d'autres congrès ethniques tenus en Russie, a été organisé non point avec des fonds publics, le financement ayant été assuré par des sponsors, et de nombreux délégués s'y sont rendus sur leurs fonds propres. En même temps, ce Congrès a rassemblé près d'une centaine de délégués des oblasti d'Arkhangelsk et de Murmansk, de l'okrug autonome nenets et d'autres régions de la Fédération de Russie. Le Congrès a adopté le Règlement sur le Conseil du peuple pomor, il en a élu les membres, et il a été proclamé «l'organe dirigeant suprême du peuple pomor ». Dans la Déclaration adoptée, il est dit :

Nous, les Pomors, peuple autochtone du nord de la Russie, ayant vécu pendant des siècles dans les oblasti d'Arkhangelsk et de Murmansk, dans la République de Carélie et dans l'okrug autonome nenets, nous proclamons notre droit à l'existence en tant que peuple autonome de la Fédération de Russie... Nous proclamons notre droit à la gestion et à l'exploitation des territoires traditionnels et des ressources naturelles au même titre que les autres peuples autochtones du Nord, inclus dans la liste unique des peuples autochtones de Russie.

La résolution du Congrès déclare que le refus, de la part des organes du pouvoir, d'inclure les Pomors dans la liste des peuples autochtones doit être vu comme une "preuve de discrimination », qui a pour but l'élimination des Pomors de l'exploitation traditionnelle des ressources naturelles.

\section{Le problème du statut}

Dans les programmes politiques du mouvement des Komis de l'Ižma et des leaders du mouvement pomor, la revendication de l'attribution aux deux groupes du statut de "petit peuple autochtone» occupe une place centrale. Le contenu même de cette notion et sa critique conceptuelle ont été examinés de manière détaillée et approfondie dans l'article de S. Sokolovskij (Sokolovskij, 2007). C'est pourquoi nous nous limiterons à rappeler que la Liste unique des petits peuples autochtones a été approuvée par le gouvernement de la Fédération de Russie en 2000 et inclut quarante peuples et groupes 
ethniques. L'attribution à un groupe de ce statut implique le soutien gouvernemental concernant l'attribution de droits prioritaires à l'exploitation des ressources foncières, l'exonération d'impôts sur le droit d'utilisation de ces ressources, l'attribution de privilèges sur la pêche du poisson et la chasse aux mammifères marins, l'application aux enfants des «peuples autochtones » de places subventionnées dans les universités, le droit à un service militaire alternatif, etc.

Le problème du statut est perçu de manière particulièrement aiguë dans la mesure où les Komis et les Pomors d'un côté, les Sames, les Nenets et les Khantys de l'autre non seulement sont voisins, mais souvent vivent dans les mêmes villages, pratiquent les mêmes activités de subsistance et rencontrent les mêmes problèmes. En même temps, certains ont un appui de la part de l'État, d'autres non. Ainsi dans la péninsule de Kola, des Sames et des Komis vivent dans les villages de Lovozero, de Krasnoščel'je et d'autres, et une partie d'entre eux sont éleveurs de rennes. Aujourd'hui, parmi les Sames, ils sont trente à travailler dans l'élevage du renne, alors que les Komis sont quatre-vingt-dix. Dans le rajon du Mezen' de l'oblast' d'Arkhangelsk, les Nenets vivent avec les Pomors dans les villages de Ruč'i, de Dolgoščel'je, de Kojda, de Majda ; en même temps ils sont exonérés d'impôts sur l'utilisation des pâturages, alors que les unités productives du rajon ont été contraintes de compter dans le budget des paiements obligatoires pour la terre. Plus encore, les Nenets et les Sames, en tant que peuples autochtones, bénéficient de quotas pour la pêche en mer, alors qu'historiquement cette activité concerne exclusivement les Pomors, puisque les Sames et les Nenets ne pêchaient que dans les rivières et dans les lacs. Les quotas pour la pêche en mer ne sont jamais utilisés pleinement, et sont souvent vendus par les leaders nenets et sames. En même temps, les quotas alloués aux Pomors sont insuffisants et ils doivent payer pour les obtenir, de même qu'ils doivent payer pour un certain volume de pêche. Un exemple typique : en 2008, les Sames de Kola se sont vu attribuer un quota de pêche à la morue de 250 tonnes. Ce quota était censé être racheté aux Sames par le responsable de la coopérative pomor de pêche Belomor, S. Samojlov, dans la mesure où ses bateaux, avec leur quota spécifique, avaient de quoi travailler seulement pour un mois. Il comptait à l'aide du quota same arriver à prolonger le travail de ses bateaux d'un mois et demi. Mais les leaders sames refusèrent le prix de cinq mille roubles par tonne de quota, ce qui était le prix qu'ils en avaient reçu l'année précédente, et ils demandèrent davantage. L'affaire ne fut pas conclue. La situation des entreprises de pêche du Mezen' est très semblable à celle des entreprises analogues sur les rives caréliennes de la mer blanche, qui relèvent de la Pomorie historique (Gnetnev, 2008, pp. 200-209).

Le problème du statut est devenu particulièrement d'actualité dans les dernières années, car par exemple, les exploitations du rajon du Mezen', qui avant avaient un troupeau de rennes, non seulement ont dû renoncer à l'élevage du renne dans les années 1990, mais, dans les nouvelles conditions, elles ont été obligées de payer pour utiliser les parcelles de terre et de toundra, alors que les Nenets de l'okrug autonome nenets étaient exonérés de cette taxe. La même situation se retrouve non seulement dans la pêche en mer, qui aujourd'hui n'est pas seulement limitée, mais mise pratiquement hors la loi, mais aussi pour les terrains de pêche fluviaux et lacustres. Dans le Nord, il s'est établi historiquement un partage des tâches entre Nenets et Pomors, qui a en même temps débouché sur différentes formes de coopération (Davydov 2006). L'ancien partage des tâches entre Nenets et Pomors a été ainsi détruit, de même qu'a été détruit l'ancien positionnement culturel des groupes ethniques, ce 
qui provoque divers conflits entre la population éponyme de l'okrug autonome nenets et la population vivant traditionnellement dans l'oblast' d'Arkhangelsk.

À Arkhangelsk, de nombreux spécialistes et certains fonctionnaires sont prêts à soutenir la revendication d'attribution aux Pomors d'un statut particulier. L'administration de l'oblast' a envoyé à deux reprises des demandes officielles au ministère du Développement régional de la Fédération de Russie demandant de soutenir les revendications des Pomors et de les inclure dans la liste unique des petits peuples autochtones. Mais comme ces demandes ont suscité des rapports négatifs des ethnologues de Moscou et de Saint-Pétersbourg, les revendications n'ont pas été satisfaites.

81 Le 9 août 2005, le jour des peuples autochtones du monde entier, l'Autonomie ethnique et culturelle des Pomors d'Arkhangelsk a diffusé un communiqué de presse annonçant que conformément aux données d'une enquête sociologique réalisée par le Centre de Recherches sociologiques et de marché «Foris », $74 \%$ de la population d'Arkhangelsk soutient l'idée de reconnaissance aux Pomors des droits des petits peuples autochtones du Nord (Press-reliz, 2005). Bien qu'à notre avis l'échantillon sur lequel a été réalisée cette enquête soit clairement insuffisant pour avoir une appréciation indubitable de l'attitude des habitants de l'oblast' d'Arkhangelsk, on peut cependant parler d'un soutien considérable accordé au mouvement pomor et aux idées qu'il promeut.

Fin avril 2007, une lettre ouverte a été envoyée à Dmitrij Medvedev, premier ministre du gouvernement de la Fédération de Russie, au nom de l'Autonomie ethnique et culturelle des Pomors de la ville d'Arkhangelsk. Les auteurs, prenant la parole au nom de tous les Pomors, demandent de l'aide "aux autorités fédérales pour démêler les problèmes de mise en œuvre de la législation russe en vigueur et des accords internationaux sur les questions nationales». La lettre souligne que «les Pomors ont été confrontés à l'impossibilité de mettre en œuvre les droits à l'auto-identification ethnique " garantis par la constitution de la Fédération de Russie et par la législation russe. Ces mêmes sujets ont été discutés au Congrès des Pomors qui a eu lieu en septembre 2007 à Arkhangelsk.

L'adresse au Premier ministre déclarait également que l'administration d'Arkhangelsk avait à deux reprises envoyé des demandes en ce sens au ministère du Développement régional, mais que les mesures nécessaires n'avaient pas été prises. Au lieu de mesures concrètes de soutien aux Pomors, les autorités fédérales avaient présenté des certificats de l'Institut d'ethnologie et d'anthropologie de l'Académie des sciences de Russie et de la «Kunstkamera» qui niaient l'existence d'une ethnie pomore à part entière. Les auteurs de la lettre demandent de montrer au ministère du Développement régional qu'il était inadmissible de prendre des décisions concernant la reconnaissance de l'existence ou de la non-existence dans le pays de communautés ethniques sans tenir compte de l'opinion de celles-ci. Le refus de reconnaître les Pomors parmi les petits peuples autochtones du Nord est qualifié de non motivé et, fort logiquement, il est fait référence à l'exemple des Kamtchadals, qui ont été inclus dans la liste des petits peuples autochtones approuvée par le gouvernement de la Fédération de Russie, alors même que les ethnographes les considèrent comme un groupe ethnographique russe. L'autonomie ethnique et culturelle des Pomors demande d'examiner la question pomore dans une commission indépendante, au travail de laquelle il est indispensable d'associer les Pomors eux-mêmes (Šabaev, 2007). 

indispensable avant tout aux habitants des zones rurales qui suivent un mode de vie et une économie traditionnels, notamment dans les villages côtiers et dans les villages du fin fond de l'oblast', où la population vit dans un système d'économie de subsistance (potagers et lopins de terre, chasse, pêche).

86

Lattribution d'un statut particulier aux Pomors pourrait apporter un stimulant complémentaire aux villages en dépression et à tous les rajony côtiers pour leur développement. Dans la mesure où les "initiatives humanitaires " d'Arkhangelsk (Šabaev, 2005b) destinées à stimuler l'initiative locale et l'esprit d'entreprise de la population rurale ont été un échec, la réforme municipale lancée par le centre fédéral n'a pas été suffisamment développée et le système des fermes n'a pas pu dans les faits se développer, dans la mesure où les habitants des campagnes aujourd'hui n'ont pratiquement pas les ressources suffisantes non seulement pour développer leur exploitation, mais souvent même pour survivre.

La spécificité de la «lutte» des Komis de l'Ižma pour un statut particulier tient non seulement à l'importance de l'élevage du renne pour l'économie locale, mais aussi à la perception de plus en plus enracinée que les éleveurs de rennes représentent les petits peuples autochtones du Nord. Mais il existe également d'autres facteurs. En effet, pour les militants komis de l'Ižma il est particulièrement important qu'ils aient été assimilés aux peuples autochtones. Conformément au décret du gouvernement de la Fédération de Russie «Sur la liste des zones d'habitat des petits peuples du Nord» du 11 janvier $1993 \mathrm{n}^{\circ} 22$, le rajon d'Ǐma de la République komie a été compté parmi les territoires sur lesquels vivent des petits peuples. Les autorités républicaines à l'époque ont considéré cette décision comme étant parfaitement légale et n'ont pas posé d'objections à l'attribution d'un statut particulier uniquement à un groupe de Komis. Cela n'a posé aucun problème sérieux non plus au sein du mouvement komi.

88 Aujourd'hui, la situation a changé et en partie, apparemment, parce que la lutte pour les ressources et pour la répartition des territoires s'est renforcée. Pratiquement partout dans le nord de la Fédération de Russie, les terrains traditionnels de chasse et de pêche sont utilisés par une population qui n'est pas marquée comme " petit peuple autochtone » en violation de la loi fédérale. En même temps, l'utilisation de ces terrains est indispensable à la survie et est justifiée du point de vue de la morale traditionnelle, dans la mesure où elle s'appuie sur des stéréotypes culturels et écologiques et sur le droit coutumier.

\section{Conclusion}

89 C'est l'État qui, avec sa politique, a provoqué le processus de réidentification, de construction de nouvelles identités ethniques. Le soutien électoral aux groupes culturels locaux et à des communautés ethniques entières, réalisé sur des critères formels (effectifs du groupe, présence en son sein d'individus pratiquant la chasse et la pêche), crée des situations conflictuelles et suscite l'aspiration à une autodétermination culturelle là où avant dominait l'aspiration à l'intégration aux groupes dominants.

On voit bien que ce qui se trouve à l'origine des processus de réidentification et de construction de l'ethnicité, c'est l'aspiration de groupes locaux à trouver des moyens d'adaptation à des conditions sociales et économiques d'existence qui ont radicalement 
changé à la fin du xxe siècle. La mémoire historique et l'ethnicité sont la ressource que les leaders locaux ont considérée comme étant la plus efficace pour adapter les groupes locaux à de nouvelles conditions de vie.

L'analyse de la situation qui s'est créée autour des Pomors et des Komis de l'Ižma nous amène à prendre en considération deux scénarios culturels différents, dont la réalisation implique la réidentification de communautés culturelles locales, qui ont existé jadis sur le territoire du Nord européen de la Fédération de Russie et qui avaient subi au $\mathrm{Xx}^{\mathrm{e}}$ siècle une érosion culturelle.

Dans le cas des Komis de l'Ižma, effectivement, on peut suivre le processus de réidentification, qui s'accompagne des tentatives de repenser l'identité du groupe et d'actualiser la mémoire historique. Mais le sens principal derrière ce processus n'est guère une distanciation culturelle du groupe par rapport à son environnement ethnique, mais l'utilisation de l'ethnicité comme forme de protection pour les intérêts du groupe et comme stimulant pour son développement socio-économique. C'est pourquoi l'accent est mis sur les particularités de la vie économique des Komis de l'Ižma, l'importance de l'environnement naturel pour la réussite économique du groupe et l'importance de la politique écologique du pays.

Dans le deuxième cas, la réidentification cache un processus de construction d'ethnicité et l'aspiration à utiliser la mémoire historique pour consolider le groupe en tant que communauté culturelle entièrement neuve. Cette construction repose sur des mythes historiques et culturels, faisant référence à l'origine du groupe et à sa mentalité sui generis. Les leaders du groupe prêtent une attention toute particulière à reconstituer et actualiser les symboles culturels - les fêtes pomores du Nouvel An, la foire de SainteMarguerite, les fêtes folkloriques, etc.

Dans le premier cas, ce qui se réalise est un projet profondément rural, puisque l'identité komie de l'Ižma est principalement soutenue par ceux qui sont directement liés à la terre. Les leaders Komis de l'Ižma eux-mêmes vivent dans des zones rurales et le leader actuel d'«Iz'vatas", Valentina Anufrieva, après son élection comme présidente, a quitté la capitale de la République komie, Syktyvkar, et est allée s'installer à Mohča, un village de la région d'Ižma.

Dans le deuxième cas, nous avons affaire à un "projet urbain ", car non seulement la plupart des habitants de l'oblast' d'Arkhangelsk qui se sont dits pomors au moment du recensement de 2002 étaient des urbains, mais ce sont aussi des intellectuels urbains, profondément intégrés dans le milieu culturel dominant. Une seule exception peut être relevée, le président du Conseil des anciens Pomors, S. Samojlov. Ce n'est pas un hasard si les leaders du mouvement accordent une attention particulière aux éléments symboliques de la culture pomore, au caractère de la perception des Pomors, alors que le problème du soutien aux pratiques de chasse et de pêche traditionnelles, bien qu'il soit présent en permanence, n'apparaît pas comme central, ni dans l'idéologie du mouvement ni dans ses programmes politiques. En tout cas, le mouvement n'a pas élaboré une stratégie détaillée et profondément argumentée de maintien et de développement des exploitations de pêche (dans les villages pomors). Cette faiblesse du mouvement a été notée par ses opposants, qui l'exploitent activement dans la polémique qui éclate régulièrement dans l'espace d'information de l'oblast' d'Arkhangelsk. 
Les deux scénarios culturels dont il a été question ici sont toujours en cours d'actualisation et il est difficile de dire lequel des deux ira jusqu'au bout et jusqu'à quel point il sera couronné de succès. Mais on peut affirmer sans risque d'erreur que ces processus ne manqueront pas de laisser des traces dans le développement culturel des régions, dans lesquelles ces scénarios, ces phénomènes culturels sont mis en œuvre.

\section{BIBLIOGRAPHIE}

ANUFRIEV 2008 = АНУФРИЕВ В.В., РУССКИЕ ПОМОРЫ. КУЛЬТУРНО-ИСТОРИЧЕСКАЯ ИДЕНТИЧНОСТЬ, АРХАНГЕЛЬСК.

ANUFRIEVA 2007 = АНУФРИЕВА В. «ИЖЕМЦЫ - ЭТО ОТДЕЛЬНАЯ ЭТНИЧЕСКАЯ ОБЩНОСТЬ», ИЗЬВАТАС, №2, 5/2007.

BERNŠTAM 1978 = БЕРНШТАМ Т.А., ПОМОРЫ: ФОРМИРОВАНИЕ ГРУППЫ И СИСТЕМА ХОЗЯЙСТВА, ЛЕНИНГРАД. .

BULATOV 1999 = БУЛАТОВ ВЛАДИМИР, РУССКИЙ СЕВЕР. КНИГА ТРЕТЬЯ. ПОМОРЬЕ (XV- НАЧАЛО XVIII В), АРХАНГЕЛЬСК.

DANILOVA, Jakov 2000 = ДАНИЛОВА Е.Н., ЯДОВ В.А., «НЕСТАБИЛЬНАЯ СОЦИАЛЬНАЯ ИДЕНТИЧНОСТЬ КАК НОРМА СОВРЕМЕННЫХ ОБЩЕСТВ», СОЦИОЛОГИЧЕСКИЕ ИССЛЕДОВАНИЯ, №10/2000.

DAVYDOV 2006 = ДАВЫДОВ А.Н., «ЭТНОХАБИТАТ НА КРАЮ ОЙКУМЕНЫ : НЕНЦЫ ОСТРОВА КОЛГУЕВ» МЕЖЭТНИЧЕСКИЕ ВЗАИМОДЕЙСТВИЯ И СОЦИОКУЛЬТУРНАЯ АДАПТАЦИЯ НАРОДОВ СЕВЕРА РОССИИ, МОсКВА.

ESIPOV 2006 = ЕСИПОВ ПАВЕЛ, «ДВОЙНЫЕ СТАНДАРТЫ», ЕДИНЫЙ МИР, ВЫПУСК 8(20) 7/2006.

FAIRCLOUGH Norman 2003, Analysing Discourse: Textual Analysis for Social Research, London.

FILATOV 2002 = ФИЛАТОВ С.Б., «АРХАНГЕЛЬСКИЙ КРАЙ - ХРАНИТЕЛЬ ДУХОВНЫХ ТРАДИЦИЙ НОВГОРОДСКОЙ РЕСПУБЛИКИ», ГУМАНИТАРНЫЕ ИССЛЕДОВАНИЯ И ГУМАНИТАРНОЕ ОБРАЗОВАНИЕ НА ЕВРОПЕЙСКОМ СЕВЕРЕ, СБОРНИК МАТЕРИАЛОВ МЕЖДУНАРОДНОЙ НАУЧНОЙ КОНФЕРЕНЦИИ (АРХАНГЕЛЬСК, 16-18 СЕНТЯБРЯ 2002 Г.). АРХАНГЕЛЬСК, С. 65.

FILIPPOV 1991 = ФИЛИППОВ Г., «ОТ СЪЕЗДА К СЪЕЗДУ», НОВЫЙ СЕВЕР, 4/6/1991 Г.

GEMP 2004 = ГЕМП КСЕНИЯ, СКАЗ О БЕЛОМОРЬЕ. СЛОВАРЬ ПОМОРСКИХ РЕЧЕНИЙ, МОСКВА-

АРХАНГЕЛЬСК.

GNETEV 2008 = ГНЕТНЕВ КОНСТАНТИН, «СОРОКА - БЕЛОМОРСК : В ОКЕАНЕ ЖИВОЙ ИСТОРИИ», CEBEP, №7+8. 2008.

HATANZEJSКIJ 2000 = ХАТАНЗЕЙСКИЙ Н.К., ДВИЖЕНИЕ «ИЗЬВАТАС»: ЕГО ДЕЛА И ЗАБОТЫ, ИЖМА.

ITOGI $2004=$ ИТОГИ ВСЕРОССИЙСКОЙ ПЕРЕПИСИ НАСЕЛЕНИЯ 2002 ГОДА. Т.4. НАЦИОНАЛЬНЫЙ СОСТАВ И ВЛАДЕНИЕ ЯЗЫКАМИ, ГРАЖДАНСТВО. КНИГА 1., МОСКВА. 
KONAKOV, KOTOV 1991 = КОНАКОВ Н.Д., КОТОВ О.В., ЭТНОАРЕАЛЬНЫЕ ГРУППЫ КОМИ: ФОРМИРОВАНИЕ И СОВРЕМЕННОЕ ЭТНОКУЛЬТУРНОЕ СОСТОЯНИЕ, МОСКВА.

KOTOV, ROGAČEV, ŠABAEV 1996 = КОТОВ О.В., РОГАЧЕВ М.Б., ШАБАЕВ Ю.П., СОВРЕМЕННЫЕ КОМИ, ЕКАТЕРИНБУРГ.

Kriničnaja 1991 = КРИНИЧНАЯ Н.А. ПРЕДАНИЯ РУССКОГО СЕВЕРА, МОСКВА.

LAŠUK 1960 = ЛАШУК Л.П., «ПРИНЦИПЫ ИСТОРИКО-ЭТНОГРАФИЧЕСКОГО РАЙОНИРОВАНИЯ КОМИ АССР», ИЗВ. КОМИ ФИЛИАЛА ВГО, СЫКТЫВКАР, 1960. ВЫП.6, с. 97-105.

LEBEDEVA 1997 = ЛЕБЕДЕВА НАДЕЖДА, «РОЛЬ КУЛЬТУРНЫХ ДИСТАНЦИЙ В ФОРМИРОВАНИИ НОВЫХ ИДЕНТИЧНОСТЕЙ», ИДЕНТИЧНОСТЬ И КОНФЛИКТ В ПОСТСОВЕТСКИХ ГОСУДАРСТВАХ, МОСКВА.

LISNIČENKO, LISNIČENKO 2007 = ЛИСНИЧЕНКО В.В., ЛИСНИЧЕНКО Н.Б. ЭКОЛОГИЯ ПОМОРА, АРХАНГЕЛЬСК.

MEZАК 2004 = МЕЗАК ЭРНЕСТ, « ИЖЕМЦЫ ОКАЗАЛИСЬ В БЕДНЫХ РОДСТВЕННИКАХ У ЯЗЬВИНЦЕВ », ЗЫРЯНСКАЯ ЖИЗНЬ, 9/8/2004.

MOSEEV 2005 = МОСЕЕВ И.И., ПОМОРСКА ГОВОРЯ. КРАТКИЙ СЛОВАРЬ ПОМОРСКОГО ЯЗЫКА, АРХАНГЕЛЬСК.

PERVAJA 1905 = ПЕРВАЯ ВСЕОБЩАЯ ПЕРЕПИСЬ НАСЕЛЕНИЯ РОССИЙСКОЙ ИМПЕРИИ 1897 Г. ПОД РЕД. Н.А. ТРОЙНИЦКОГО. Т. ІІ. ОБЩИЙ СВОД ПО ИМПЕРИИ РЕЗУЛЬТАТОВ РАЗРАБОТКИ ДАННЫХ ПЕРВОЙ ВСЕОБЩЕЙ ПЕРЕПИСИ НАСЕЛЕНИЯ, ПРОИЗВЕДЕННОЙ 28 ЯНВАРЯ 1897 ГОДА., САНКТ-ПЕТЕРБУРГ. PRESS-RELIZ 2005 = «ПРЕСС-РЕЛИЗ НКА ПОМОРОВ 9 АВГУСТА 2005 Г.», ИНФОРМАЦИОННЫЙ ЦЕНТР ФИННО-УГОРСКИХ НАРОДОВ.

RUSSKAJA 1989 = РУССКАЯ ДИАЛЕКТОЛОГИЯ, ПОД РЕД. Л.Л.КАСАТКИНА, МОСКВА.

RUŽNIKOV 2005 = РУЖНИКОВ А.В., «ПОМОРСКОЕ СУДОСТРОЕНИЕ И МОРЕПЛАВАНИЕ В ХІХ - НАЧАЛЕ ХХ ВЕКОВ: ТЕНДЕНЦИИ РАЗВИТИЯ», ЛОДИЯ. СБОРНИК СТАТЕЙ. №1, АРХАНГЕЛЬСК.

ŠABAEV 2003 = ШАБАЕВ Ю.П., «КОМУ НУЖНЫ ПОМОРЫ?», СЕТЬ ЭТНОЛОГИЧЕСКОГО МОНИТОРИНГА И РАННЕГО ПРЕДУПРЕЖДЕНИЯ КОНФЛИКТОВ. БЮЛЛЕТЕНЬ №51, 2003.

ŠABAEV 2005 = ШАБАЕВ Ю.П., «ЭТНОДЕМОГРАФИЧЕСКОЕ РАЗВИТИЕ КОМИ В КОНТЕКСТЕ ДЕМОГРАФИЧЕСКИХ ПРОЦЕССОВ У ФИННО-УГОРСКИХ НАРОДОВ РОССИЙСКОЙ ФЕДЕРАЦИИ», ДОКЛАД НА ПЛЕНАРНОМ ЗАСЕДАНИИ ВСЕРОССИЙСКОЙ НАУЧНОЙ КОНФЕРЕНЦИИ ПО ИСТОРИЧЕСКОЙ ДЕМОГРАФИИ «ЭТНОДЕМОГРАФИЧЕСКИЕ ПРОЦЕССЫ НА СЕВЕРЕ ЕВРАЗИИ (XI - XX Вв.)». СЫКТЫВКАР.

ŠABAEV 2005b = ШАБАЕВ Ю.П., «АРХАНГЕЛЬСКАЯ ОБЛАСТЬ», ЭТНИЧЕСКАЯ СИТУАЦИЯ И КОНФЛИКТЫ В СТРАНАХ СНГ И БАЛТИИ. ЕЖЕГОДНЫЙ ДОКЛАД, 2004, МОСКВА.

ŠABAEV 2007 = ШАБАЕВ Ю.П., «СПОР О ПОМОРАХ: КТО ПРАВ?», БЮЛЛЕТЕНЬ СЕТИ ЭТНОЛОГИЧЕСКОГО МОНИТОРИНГА И РАННЕГО ПРЕДУПРЕЖДЕНИЯ КОНФЛИКТОВ, №73 МАЙ-ИЮНЬ 2007.

SIVKOVA 2003 = СИВКОВА АННА, «ИЖЕМЦЫ ПРОСЯТСЯ В МАЛОЧИСЛЕННЫЕ», РЕСПУБЛИКА, $18 / 7 / 2003$.

SIVКOVA 2008 = СИВКОВА АННА, «КОРЕННОЙ НАРОД ДОЛЖЕН ОСТАТЬСЯ ЕДИНЫМ», РЕСПУБЛИКА, $8 / 7 / 2008$ г.

SMETANIN 2005 = СМЕТАНИН АЛЕКСАНДР, «ЧУЖИЕ В СВОЕЙ РЕСПУБЛИКЕ», БУДНИ, 14/4/2005. 
ŠNIRELMAN 2000 = ШНИРЕЛЬМАН В., «ЦЕННОСТЬ ПРОШЛОГО: ЭТНОЦЕНТРИСТСКИЕ ИСТОРИЧЕСКИЕ МИФЫ, ИДЕНТИЧНОСТЬ И ЭТНОПОЛИТИКА», РЕАЛЬНОСТЬ ЭТНИЧЕСКИХ МИФОВ. АНАЛИТИЧЕСКАЯ СЕРИЯ. ВЫПУСК 3, МОСКВА.

SOKOLOVSKIJ 2001 = СОКОЛОВСКИЙ С.В., «ПРОБЛЕМЫ САМООПРЕДЕЛЕНИЯ И САМОУПРАВЛЕНИЯ КОРЕННЫХ НАРОДОВ», in СОКОЛОВСКИЙ С.В., ОБРАЗЫ ДРУГИХ В РОССИЙСКИХ НАУКЕ, ПОЛИТИКЕ И ПРАВЕ, МОСКВА.

SOKOLOVSKIJ 2004 = СОКОЛОВСКИЙ С.В., ПЕРСПЕКТИВЫ РАЗВИТИЯ КОНЦЕПЦИИ ЭТНОНАЦИОНАЛЬНОЙ ПОЛИТИКИ В РОССИЙСКОЙ ФЕДЕРАЦИИ, МОСКВА.

SOKOLOVSKIJ 2007 = СОКОЛОВСКИЙ С.В., «ПРАВОВОЙ СТАТУС И ИДЕНТИЧНОСТЬ КОРЕННЫХ НАРОДОВ (ПО МАТЕРИАЛАМ ВСЕРОССИЙСКОЙ ПЕРЕПИСИ НАСЕЛЕНИЯ 2002 ГОДА)», РАСЫ И НАРОДЫ, СОВРЕМЕННЫЕ ЭТНИЧЕСКИЕ И РАСОВЫЕ ПРОБЛЕМЫ. ЕЖЕГОДНИК. ВЫП. 33. МОСКВА.

SzTOMPKA P., 2004, "The Trauma of Social Change: A Case of Postcommunist Societies", in Alexander J.C.,Eyerman R., GIESEn B., SMELSER N.J., SZTOMPKA P. (eds.), Cultural Trauma and Collective Identity, Dercley, CA: University of California Press

TEREBIHIN 2004 = ТЕРЕБИХИН Н.М., МЕТАФИЗИКА СЕВЕРА, АРХАНГЕЛЬСК.

TEREBIHIN, Nesanelis 2008 = ТЕРЕБИХИН Н.M., НЕСАНЕЛИС Д.А., «ГЕОГРАФИЧЕСКИЕ ОБРАЗЫ ЭТНОКУЛЬТУРНОГО ЛАНДШАФТА КОМИ-ЗЫРЯН», ПОМОРСКИЕ ЧТЕНИЯ ПО СЕМИОТИКЕ КУЛЬТУРЫ. САКРАЛЬНАЯ ГЕОГРАФИЯ И ТРАДИЦИОННЫЕ ЭТНОКУЛЬТУРНЫЕ ЛАНДШАФТЫ НАРОДОВ ЕВРОПЕЙСКОГО СЕВЕРА. ВЫПУСК 3. АРХАНГЕЛЬСК.

THEORIES 1996, Theories of Ethnicity. A Classical Reader, Ed. By W. Sollors, New York.

TIŠKOV 2003 = ТИШКОВ В.А., РЕКВИЕМ ПО ЭТНОСУ. ИССЛЕДОВАНИЯ ПО СОЦИАЛЬНО-КУЛЬТУРНОЙ АНТРОПОЛОГИИ. МОСКВА.

TIŠKOV 2005 = ТИШКОВ В.А. «О КУЛЬТУРНОМ МНОГООБРАЗИИ», ЭТНОГРАФИЧЕСКОЕ ОБОЗРЕНИЕ, 2005,№1.

VLASOVA 2005 = ВЛАСОВА И.В., «ЭТНИЧЕСКАЯ ИСТОРИЯ И ФОРМИРОВАНИЕ НАСЕЛЕНИЯ РУССКОГО СЕВЕРА», ЭТНОПАНОРАМА. 2005. №1-2.

VOGT H., 2005, Between Utopia and Disillusionment: A Narrative of the Political Transformation in Eastern Europe, New York: Berghahn Books.

VoRONKOv, Osvald 1998 = ВОРОНКОВ ВИКТОР, ОСВАЛЬД ИНГРИД, «ПОСТСОВЕТСКИЕ ИДЕНТИЧНОСТИ», КОНСТРУИРОВАНИЕ ЭТНИЧНОСТИ. ЭТНИЧЕСКИЕ ОБЩИНЫ САНКТ-ПЕТЕРБУРГА, САНКТ-ПЕТЕРБУРГ.

VOZVRAŠČENIE... 2008 = «ВОЗВРАЩЕНИЕ КОМИ-ИЖЕМЦАМ СТАТУСА КОРЕННОГО МАЛОЧИСЛЕННОГО НАРОДА ЛОГИЧНО И ОБОСНОВАННО», НОВЫЙ СЕВЕР, 5/7/2008 Г.

ŽEREBCOV 1982 = ЖЕРЕБЦОВ Л.Н., ИСТОРИКО-КУЛЬТУРНЫЕ ВЗАИМООТНОШЕНИЯ КОМИ С СОСЕДНИМИ НАРОДАМИ, МОСКВА.

\section{NOTES}

1. En russe : БОЛЬШЕЗЕМЕЛЬСКАЯ ТУНДРА.

2. En russe : ВЫГОВСКАЯ ПУСТЫНЬ. 
3. En russe : БЕспоповцы ; il s'agit des groupes de vieux croyants qui, à la mort de leur prêtre ordonné avant la réforme de Nikon, ont poursuivi leurs pratiques sans l'aide de prêtres.

4. КОРЕННОЙ МАЛОЧИСЛЕННИЙ НАРОД.

\section{RÉSUMÉS}

Cet article traite de processus culturels complexes : ceux qui sont en cours dans le Nord européen de la Russie, et qui tiennent à des mutations dans le mode d'auto-identification des groupes culturels locaux. Nous étudierons ici deux d'entre eux : les Pomors et les Komis de l'Ižma. On a coutume de considérer les Pomors comme un groupe ethnographique russe du Nord, le seul dont l'économie traditionnelle soit fondée sur la pêche maritime et la chasse aux mammifères marins. Les Komis de l'Ižma, quant à eux, sont le groupe le plus septentrional des Komis, donc des FinnoOugriens. Leur économie est fondée sur l'élevage du renne. Au début des années 2000, les deux groupes (dont la formation en tant que communautés culturelles autonomes remonte au $\mathrm{XVIII}^{\mathrm{e}}$ siècle, mais qui au $\mathrm{xx}^{\mathrm{e}}$ siècle avaient en grande partie perdu leur identité) traversent une période de réidentification. On examinera les raisons et les conditions dans lesquelles ces processus se déroulent, ainsi que le contenu de la nouvelle identité de ces groupes, qui diffère considérablement des formes antérieures. Les idéologues du mouvement pomor ont tendance à considérer les Pomors non pas comme des Slaves, mais comme des Finno-Ougriens, alors que les leaders du mouvement de l'Ižma considèrent que les Iz'vatas doivent être considérés comme un peuple à part et non pas comme une partie de la communauté ethnique des Komis.

In this article, the author analyses the complex cultural processes at work in Northern Russia connected to changes in the identification of local groups. The article is focused on two groups: the Pomors and the Izhma Komi. The Pomors are usually considered a Northern Russian ethnographic group, the only one to have maintained an economy based on sea fishing and sea mammal hunting. The Izhma Komi are the northernmost group of Komi, whose economy is based on reindeer herding: they are thus Finno-Ugrians. From the beginning of the 2000s, both groups (who were constituted as autonomous cultural communities in the 18th century, but whose identity faded somehow in the 20th) have been living a reidentification process. This article analyses both the reasons for and conditions of this change, as well as the contents of the new identities of the groups, which definitely differs from the previous forms. The Pomors' movement ideologists tend to consider that the Pomors are not Slavs, but Finno-Ugrians, while the leaders of the Izhma movement assert that the iz'vatas are to be seen as a separate people, and not a part of the Komi ethnic community.

СТАТЬЯ ПОСВЯЩЕНА АНАЛИЗУ СЛОЖНЫХ КУЛЬТУРНЫХ ПРОЦЕССОВ, КОТОРЫЕ ПРОИСХОДЯТ НА ЕВРОПЕЙСКОМ СЕВЕРЕ РОССИИ И СВЯЗАНЫ С ИЗМЕНЕНИЯМИ ХАРАКТЕРА ИДЕНТИФИКАЦИИ У ЛОКАЛЬНЫХ КУЛЬТУРНЫХ ГРУПП. ОБЪЕКТАМИ ИЗУЧЕНИЯ ЯВЛЯЮТСЯ ДВЕ ГРУППЫ : ПОМОРЫ И КОМИ-ИЖЕМЦЫ. ПОМОРОВ ПРИНЯТО СЧИТАТЬ СЕВЕРНОЙ ЭТНОГРАФИЧЕСКОЙ ГРУППОЙ РУССКИХ - ЕДИНСТВЕННОЙ ГРУППОЙ, ТРАДИЦИОННОЕ ХОЗЯЙСТВО КОТОРОЙ ОСНОВЫВАЛОСЬ НА МОРСКОМ РЫБОЛОВНОМ И ЗВЕРОБОЙНОМ ПРОМЫСЛЕ. КОМИ-ИЖЕМЦЫ - САМАЯ СЕВЕРНАЯ ГРУППА КОМИ, ОСНОВУ ХОЗЯЙСТВА КОТОРЫХ СОСТАВЛЯЛО ТОВАРНОЕ ОЛЕНЕВОДСТВО, Т.Е. ОНИ ОТНОСЯТСЯ К ФИННО-УГРАМ. С НАЧАЛА 2000-Х ГОДОВ ОБЕ ГРУППЫ (КОТОРЫЕ СЛОЖИЛИСЬ КАК САМОСТОЯТЕЛЬНЫЕ КУЛЬТУРНЫЕ СООБЩЕСТВА К XVIII В., А В XX В. В ЗНАЧИТЕЛЬНОЙ МЕРЕ 
УТРАТИЛИ СВОЮ ИДЕНТИЧНОСТЬ) ПЕРЕЖИВАЮТ ПРОЦЕССЫ РЕИДЕНТИФИКАЦИИ. РАССМАТРИВАЮТСЯ ПРИЧИНЫ И УСЛОВИЯ, В КОТОРЫХ ПРОТЕКАЮТ НАЗВАННЫЕ ПРОЦЕССЫ, А ТАКЖЕ СОДЕРЖАНИЕ НОВОЙ ИДЕНТИЧНОСТИ ГРУПП, КОТОРОЕ СУЩЕСТВЕННО ОТЛИЧАЕТСЯ ОТ ПРЕЖНИХ ФОРМ. ИДЕОЛОГИ ПОМОРСКОГО ДВИЖЕНИЯ СКЛОННЫ СЧИТАТЬ ПОМОРОВ НЕ СЛАВЯНАМИ, А ФИННО-УГРАМИ, А ЛИДЕРЫ ИЖЕМСКОГО ДВИЖЕНИЯ НАСТАИВАЮТ, ЧТО КОМИИЖЕМЦЕВ НАДО РАССМАТРИВАТЬ КАК ОТДЕЛЬНЫЙ НАРОД, А НЕ КАК ЧАСТЬ ЭТНИЧЕСКОГО СООБЩЕСТВА КОМИ.

\section{INDEX}

nomsmotscles Sames, Khantys, Komis de l'Ižma, Oudmourtes, Bessermans, Mordves, Erzas, Mokchas, Tatars, Nénetses, Mišars, Tatars de Sibérie, Russes, Cosaques, Kamtchadales, Caréliens motsclesru ЭТНИЧЕСКОЕ САМОСОЗНАНИЕ, ПЕРЕПИСЬ, ИДЕНТИЧНОСТЬ, ОЛЕНЕВОДСТВО, ИЗЬВАТАС, ПОМОРЫ, СТАТУС, АРХАНГЕЛЬСКИЙ КРАЙ, КОМИ РЕСПУБЛИКА, СОВЕТСКОЕ ВРЕМЯ, ПОСТСОВЕТСКОЕ ВРЕМЯ, СОЦИОЛОГИЯ

Thèmes : sociologie

Index géographique : région d'Arkhangelsk, Komi (République), Moscou, Novgorod, Oural, Arhangel'sk (oblast'), Carélie (République), Dvina septentrionale, Ežva, Lovozero, Kanin, Mezen', Murmansk, Olonets, Onega, Pomor'e, Ruč'i, Dolgoščel'je, Kojda, Majda, Saint-Pétersbourg, Severodvinsk, Vologda, Vyg, Ižma, Bakur, Gam, Mohča, Mošjuga, Sizjabsk, Pečora, Syktyvkar, Udora, Ust'-Cil'ma, Nouvelle-Zemble (île), Sibérie occidentale, Ob' (rivière), Norvège, Spitzberg Mots-clés : Daniliens, conscience ethnique, élevage du renne, Komis de l'Ižma Iz'vatas, identité, Komis, Pomors, mouvement national, Vieux croyants, recensement

Index chronologique : XXe siècle

Keywords : ethnical awareness, Census, identity, reindeer herding, national movement, Iz'vatas, Pomors, status, Arkhangelsk region, Komi Republic, Soviet period, post-soviet period, sociology 